Example. Let $C$ be the set of all continuous real valued functions on the unit interval. We assume that $C$ is metrized by the usual uniform metric. We define a function $f$ on $C$ by letting

$$
f(u)=\{t \mid u(t)>0\} \quad \text { for each } \quad u \in C .
$$

It is easy to verify that $f$ is continuous with respect to the $T_{1}$ topology, but that $f$ is not continuous with respect to the $T_{2}$ topology. It follows from Theorem 13 that $f$ is continuous with respect to $d$ on a residual subset of $C$. It is easy to see that $f$ is $d$-continuous at $u$ if and only if $\mu\{t \mid u(t)=0\}=0$.

\title{
References
}

[1] A. Alexiewicz et W. Orlicz, Sur la continuité et la classification de Baire des fonctions abstraites, Fundamenta Mathematicae 35 (1948), p. 105-126.

[2] G. Choquet, Convergences, Annales de l'Université de Grenoble 23 (1947), p. $57-112$.

[3] M. K. Fort, Jr., A unified theory of semi-continuity, Duke Mathematical Journal 16 (1949), p. 237-2:-a.

[4] - Points of continuity of semi-continuous functions, Publicationes Mathematicae 2 (1951), p. 100-102.

[5] H. Hahn, Reelle Funktionen, Leupzig 1932.

[6] J. L. Kelley, Contergenee in topology, Duke Mathematical Journal 17 (1950), p. $277-283$

[7] E. Michael, Topologies on spaces of subsets, Transactions of the American Irathematical Society 71 (1951), p. 152-182.

[8] D. Montgomery, Topological groups of differentiable transformations, Annals of Mathematics 46 (1945), p. 382-387.

[9] B. J. Pettis, On continuity and openness of homomorphisms in topological groups, Annals of Mathematics 52 (1950), p. 293-308.

UNIVERSITY OF GEORGIA

Reşu par la Rédaction le 15.9.1954

\section{Über eine \\ Dimensionstheorie in topologischen Verbänden}

von

\section{H. Ho f man n (Nürnberg)}

Die mengentheoretische Topologie hat weitgehende Verallgemeinerungen zu einer Topologie der Vereine und Verbände erfahren (Nöbeling [3] und Sikorski [4]). Insbesondere ist auch die Menger-Urysohn'sche Dimensionstheorie von R. Sikorski auf gewisse topologische Verbände ( $C$-Algebren (Sikorski [4] und [5])) übertragen worden. Allerdings verwendet Sikorski [כ̆] einen globalen Dimensionsbegriff, mit dem sich nicht alle Sätze der Punktmengen-Dimensionstheorie formulieren lassen. Die vorlirgende Arbeit hat nun zum Ziel, unter Zugrundelegung einer allgemeineren, lokalen Dimensionsdefinition eine Theorie zu entwickeln, in der noch fehlende Sätze bewiesen werden können. Es wird sich dabei zeigen, daß dieser lokale Dimensionsbegriff für $C$-Algebren, hier $S$-Verbände genannt, mit dem Sikorski'schen zusammenfällt.

Die rorliegende Arbeit stützt sich auf G. Nöbeling [3] und verwendet die dortigen Begriffe, Bezeichnungen und Sätze.

Ein für alle Mal sei ein klassisch-topologischer Boole-Verband $\mathfrak{B}$ rorgelegt.

Definition. $\mathfrak{B}$ heiße speziell ein Sikorski-Verband oder kurz ein $S$-Terband, wenn $\mathfrak{B}$ ein $\sigma$-Terband, regulär und $T_{1}$-topologisch ist und außerdem eine abzählbare Basis ${ }^{1}$ ) besitzt.

Es kann leicht gezeigt werden, daß jeder $S$-Verband eine abzählbare reguläre Basis besitzt. Ein $S$-Verband ist demnach dasselbe wie eine Sikorski'sche $C$-Algebra.

In Anlehnung an die Stoffeinteilung Mengers [2] behandelt die vorliegende Arbeit nach der Formulierung der Dimensionsdefinition (\$1) die Dimension einzelner Somen ( $\$ 2)$, Summen- und Zerspaltungssätze (§3), die lokale dimensionelle Struktur ron $\$$-Verbänden $(\$ 4)$, Überdeckungssätze ( $§ 5$ ), die Beziehungen globaler Trennungs- und Zusammen-

\footnotetext{
1) „Basis": ist immer als offene Basis gemeint.
} 
hangseigenschaften von $\mathfrak{B}$ zur Dimension von $\mathfrak{B}(\S 6)$ und das Verhalten der Dimension bei Abbildungen ( $\$$ ). Die Beweise folgen im allgemeinen den von Menger [2] und Hurewicz-Wallman [1] verwendeten Gedankengängen.

\section{§ 1. Der Dimensionbegriff}

Definition. Es sei $n$ eine ganze Zahl, $0 \leqslant n<\infty$.

(a) $\mathfrak{B}$ heiße hr̈chstens $n$-dimensional, in Zeichen $\operatorname{dim} \mathfrak{B} \leqslant n$, wenn es zu jedem Soma $S>O$ (falls ein solches vorhanden ist) und jeder Umgebung $U$ von $S$ ein offenes Soma $V \leqslant U$ mit $V \wedge S>0$ und $\operatorname{dim} \mathrm{b} V \leqslant n-1$ gibt ${ }^{2}$. Ein Soma $A$ heiße höchstens $n$-dimensional, in Zeichen $\operatorname{dim} A \leqslant n$, wenn $\mathfrak{V}_{A}$ höchstens $n$-dimensional ist ${ }^{3}$ ). Höchstens (-1)-dimensional sei das Nullsoma und nur dieses.

(b) $\mathfrak{B}$ (bzw. ein Soma $\mathcal{A}$ ) heiße mindestens n-dimensional, in Zeichen $\operatorname{dim} \mathfrak{B} \geqslant n$ (bzw. $\operatorname{dim} A \geqslant n$ ), wenn $\mathfrak{B}$ (bzw. $A$ ) nicht höchstens $(n-1)$ -dimensional ist. Mindestens (-1)-dimensional heiße jedes Soma.

(c) $\mathfrak{B}$ (bzw. ein Soma $A$ ) heiße (genau) $n$-dimensional, in Zeichen $\operatorname{dim} \mathfrak{B}=n(\mathrm{bzw} . \operatorname{dim} A=n)$, wenn $\mathfrak{B}$ (bzw. $A$ ) zugleich höchstens $n$-dimensional und mindestens $n$-dimensional ist.

(d) Gilt für keine endliche Zahl $n \operatorname{dim} \mathfrak{B} \leqslant n($ bzw. $\operatorname{dim} A \leqslant n)$, so werde $\operatorname{dim} \mathfrak{B}=\infty$ (bzw. $\operatorname{dim} A=\infty$ ) geschrieben.

In einem topologischen Raum $\Re$, aufgefaßt als Verband $\mathfrak{B}$ (Nöbeling [3]) ist diese Definition mit der Dimensionsdefinition von Menger- Urysohn äquivalent.

Eine andere Formulierung der Dimensionsdefinition wird durch den folgenden Satz angegeben.

1.1. Voraussetzung: $0 \leqslant n<\infty$.

Behauptung: Es ist dann und nur dann $\operatorname{dim} \mathfrak{B} \leqslant n$, wenn es eine Basis $\mathfrak{B}^{\prime}$ von $\mathfrak{B}$ gibt, deren Somen höchstens $(n-1)$-dimensionale Begrenzungen haben.

Beweis. (a) Dann. Ist die Bedingung des Satzes erfüllt und ist ein Soma $S>O$ sowie eine Umgebung $U$ von $S$ vorgelegt, so ist $U$ als offenes Soma die Vereinigung von Somen der Basis $\mathfrak{B}^{\prime}$. Mindestens eines dieser Somen ist zu $S$ nicht fremd. Daraus folgt $\operatorname{dim} \mathfrak{B} \leqslant n$.

\footnotetext{
2) bV bezeichnet die Begrenzung $\bar{\nabla} \wedge \mathrm{c} V$ von $\nabla$. - Es wäre denkbar, von $U$ nur wirkliche Verallgemeinerung, weil es bei auch zu jedem Soma $S>O$ und jedem bei Gültigkeit der oben formulierten Bedingung auch zu jedem Soma $S>O$ und jedem offenen Soma $U$ mit $U \wedge S>O$ ein offenes Soma $\nabla \leqslant U$ mit $V \wedge S>0$ und $\operatorname{dim} b V \leqslant n-1$ gibt: $P=U \wedge S$ ist nicht leer; $U$ ist eine Umgebung von $P$; für das zu $P$ und $U$ existierende Soma $V \leqslant U$ mit dim $b V \leqslant n-1$ gilt
$\nabla \wedge S \geqslant \nabla \wedge P>O$.

3) $\mathfrak{V}_{A}$ bedeutet den aus allen Teilsomen von $A$ bestehenden Unterverband von $\mathfrak{Y}$.
}

(b) Nur dann. Ist $\operatorname{dim} \mathfrak{B} \leqslant u$, so ist das System aller offenen Somen $\nabla$ mit $\operatorname{dim} \mathrm{b} V \leqslant n-1$ eine Basis von $\mathfrak{B}$.

Über die Mächtigkeit der in 1.1 genamnten Basis gibt der im Folgenden mehrmals benötigte Satz 1.2 Aufschluß:

1.2. Foraussetzung: $0 \leqslant n<\infty ; \operatorname{dim} \mathfrak{i} \leqslant n$.

Behauptung: Besitzt $\mathfrak{B}$ eine Basis $\mathfrak{B}$ einer Mächtigkeit $\leqslant \mathrm{m}\left(\mathrm{nt} \geqslant \aleph_{0}\right)$, so besitat $\mathfrak{B}$ auch eine Basis $\mathfrak{B}^{\prime}$ einer Ilächtigkeit $\leqslant m$ mit $\operatorname{dim} \mathrm{bW} \leqslant n-1$ für jedes $W \in \mathfrak{B}^{\prime}$.

Beweis. Für jedes Paar $\left(U_{0}, U\right)$ von Somen aus $\mathfrak{B}$, für welches $U_{0} \leqslant U$ gilt und ein offenes Soma $W$ mit $U_{0} \leqslant W \leqslant U$ und $\operatorname{dim} \mathrm{b} W \leqslant n-1$ existiert, wählen wir ein solches Soma $W$ aus; es sei $\mathfrak{B}^{\prime}$ das System aller so ausgewählten Somen. Dann hat $\mathfrak{B}^{\prime}$ zunächst eine Mächtigzeit $\leqslant \mathrm{m}$. Um zu zeigen, daß $\mathfrak{B}^{\prime}$ eine Basis ron $\mathfrak{B}$ ist, genügt es nachzuweisen, daß jedes Soma $U \in \mathfrak{B}$ die Vereinig ung von Somen $\in \mathfrak{B}^{\prime}$ ist. Angenommen, für ein Soma $U \in \mathfrak{B}$ wäre dies nicht der Fall. Dann existiert ein Soma $S$ mit $O<S \leqslant U$, das fremd ist zu allen Somen $W \leqslant U$ aus $\mathfrak{B}^{\prime}$. Wegen $\operatorname{dim} \mathfrak{B} \leqslant n$ existiert aber in $\mathfrak{B}$ ein offenes Soma $V \leqslant U$ mit $\nabla \wedge S>0$ und $\operatorname{dim} \mathrm{b} V \leqslant n-1$; in der Basis $\mathfrak{B}$ existiert weiter ein Soma $U_{0} \leqslant V$ mit $U_{0} \wedge S>0$. Folglich existiert in $\mathfrak{B}^{\prime}$ ein Soma $W$ mit $U_{0} \leqslant W \leqslant U$, also mit $W \wedge S>0$, was im Widerspruch zur Annahme steht.

\section{§ 2. Die Dimension einzelner Somen}

2.1. Voraussetzung: $A$ sei ein betiebiges Soma; $-1 \leqslant n \leqslant \infty$. Behauptung: Aus $\operatorname{dim} \mathfrak{B} \leqslant n$ folgt $\operatorname{dim} A \leqslant n$.

Beweis (Hurewicz-Wallman [1], Theorem III. 1, S. 26) (durch vollständige Induktion). Für $n=-1$ ist die Behauptung evident. Sie werde nun für $-1 \leqslant n \leqslant m-1$ als bereits bewiesen arg nnommen; ferner sei $\operatorname{dim} \mathfrak{B} \leqslant m$. Sind dann ein nicht-leeres Soma $S \leqslant A$ und eine beliebige Umgebung $U^{\prime}$ in $A$ von $S$ vorgelegt, so gibt es zunächst ein in $\mathfrak{B}$ offenes Soma $U$ mit $U^{\prime}=U \wedge A$. $U$ ist eine Dmgebung in $\mathfrak{S}$ von $S$; aus $\operatorname{dim} \mathfrak{B} \leqslant m$ folgt daher die Existenz eines in $\mathfrak{B}$ offenen Somas $\nabla \leqslant U$ mit $\nabla \wedge S^{>}>0$ und $\operatorname{dim} \mathrm{b} V \leqslant m-1$. Setzt man nun $V \wedge A=V^{\prime}$, so ist $\nabla^{\prime}$ in $A$ offen und es gilt: $V^{\prime} \leqslant U^{\prime}$, weil $V \leqslant U$ und daher $\nabla \wedge A \leqslant U \wedge A ; V^{\prime} \wedge S>0$, weil $A \wedge S=S$ and daher $\left.(\nabla \wedge A) \wedge S=V \wedge S>0 ; \quad \mathrm{b}_{A} \nabla^{\prime} \leqslant \mathrm{b} V^{4}\right)$, weil einerseits $\mathrm{b}_{A} \nabla^{\prime} \leqslant A$ und $\nabla^{\prime} \wedge \mathrm{b}_{A} \nabla^{\prime}=0$, also $V \wedge \mathrm{b}_{A} \nabla^{\prime}=O$, andererseits $\mathrm{b}_{A} \nabla^{\prime} \leqslant \overline{V^{\prime}}$, also $\mathrm{b}_{A} \nabla^{\prime} \leqslant \bar{V}$ ist. Wendet man jetzt die Induktionsvoraussetzung auf $d_{1} n$ Verband $\mathfrak{B}_{b}$ und auf das Soma $\mathrm{b}_{A} \nabla^{\prime} \in \mathfrak{B}_{\mathrm{b} V}$ an, so erhält man $\operatorname{dim} \mathrm{b}_{A} \nabla^{\prime} \leqslant m-1$. Auf Grund dieser Eigenschaften folgt aus der Existenz des Somas $\nabla^{\prime} \operatorname{dim} A \leqslant m$. Die Behauptung gilt also auch für $n=m$ und ist damit für alle endlichen Zahlen $n$ bewiesen. Für $\mathrm{n}=\infty$ ist sie evident.

\footnotetext{
4) $\mathrm{b}_{A} \Gamma^{\prime}$ bezeichnet die Begrenzung in $A$ von $V^{\prime}$.
} 
2.2. Voraussetzung: $-1 \leqslant n<\infty$.

Behauptung: Ist $\operatorname{dim} \mathfrak{B}=n$, so existiert in $\mathfrak{B}$ für jede ganize Zahl $m$ mit $-1 \leqslant m \leqslant n$ ein genau m-dimensionales Soma.

Beweis (Hurewicz-Wallman [1], S. 24, Bem. D)). Für $m=u$ ist $E$, für $m=-1$ ist $O$ das gesuchte Soma. Nun sei $-1<m<n$. Wegen $\operatorname{dim} \mathfrak{B}=n$ existiert in $\mathfrak{B}$ ein Soma $A_{1}$ (nämlich die Begrenzung $b V$ eines offenen Somas $V$ ) mit $\operatorname{dim} A_{1}=n-1$. Dann ist $\operatorname{dim} \mathfrak{B}_{1}=n-1$ für $\mathfrak{B}_{1}=\mathfrak{B}_{A_{1}}$. Die Wiederholung dieses Schrittes liefert ein Soma $A_{2} \in \mathfrak{B}_{1}$ mit $\operatorname{dim} A_{2}=n-2$. Nach $n-m$ Schritten erhält man ein Soma $A_{n-m}$ mit $\operatorname{dim} A_{n-m}=m$.

Die Dimension eines Somas $A$ ist durch Eigenschaften des Verbandes $\mathfrak{B}_{A}$ definiert. Sie kann aber statt dessen auch durch Eigenschaften von $\mathfrak{B}$ gekennzeichnet werden $\left.{ }^{5}\right)$ :

2.3. Voraussetzung: A sei ein beliebiges Soma; $0 \leqslant n<\infty$.

Behauptung: Damit $\operatorname{dim} A \leqslant n$ sei, ist hinreichend und, falls $\mathfrak{B}$ vollständig normal ist, auch notwendig, daß es zu jedem nicht-leeren Soma $S \leqslant A$ und zu jeder Umgebung $U$ von $S$ ein offenes Soma $V \leqslant U$ mit $V \wedge S>0$ und $\operatorname{dim} \mathrm{b} V \wedge A \leqslant n-1$ gibt.

Beweis (Hurewicz-Wallman [1], S. 27, Bem. A)). (a) Hinreichend. Die Bedingung des Satzes sei erfüllt; ferner seien ein nicht-leeres Soma $S \leqslant A$ and eine beliebige Umbegung $U^{\prime}$ in $A$ von $S$ vorgelegt. Da $U^{\prime}$ in $A$ offen ist, gibt es ein (in $\mathfrak{B}$ ) offenes Soma $U$ mit $U^{\prime}=U \wedge A$. Zum Soma $S$ und seiner Umgebung $U$ existiert nach Voraussetzung ein (in $\mathfrak{B}$ ) offenes Soma $V \leqslant U$ mit $V \wedge S>0$ und $\operatorname{dim} b \nabla \wedge A \leqslant n-1$. Das Soma $V^{\prime}$ $=V \wedge A$ ist dann in $A$ offen und es gilt: $\nabla^{\prime} \wedge S>0 ; V^{\prime} \leqslant U^{\prime} ; \mathrm{b}_{A} V^{\prime} \leqslant \mathrm{b} V \wedge A$, also $\operatorname{dim} \mathrm{b}_{A} V^{\prime} \leqslant n-1$ nach 2.1. Folglich ist $\operatorname{dim} A \leqslant n$.

(b) Notwendig. $\mathfrak{B}$ sei rollständig normal und es sei $\operatorname{dim} A \leqslant n$. Ferner seien ein nicht-leeres Soma $S \leqslant A$ und eine beliebige Umgebung $U$ von $S$ gegeben. Das Soma $U^{\prime}=U A A$ ist dann in $A$ offen und es ist $S \leqslant U^{\prime}$. Wegen $\operatorname{dim} A \leqslant n$ gibt es folglich ein in $A$ offenes Soma $V^{\prime} \leqslant C^{\prime}$ mit $\nabla^{\prime} \wedge S>0$ und $\operatorname{dim} b_{A} V^{\prime} \leqslant n-1$. Die beiden Somen $V^{\prime}$ und $X=A \wedge c \overline{V^{\prime}}$ sind fremd und in ihrer Vereinigung abgeschlossen. Weil $\mathfrak{B}$ vollständig normal ist, gibt es daher ein offenes Soma $W$ mit $V^{\prime} \leqslant W$ und $\bar{W} \wedge X=0$. Setzt man dann $W \wedge U=V$, so ist $V$ offen und es gilt: $V \leqslant U ; V \wedge S>0$, weil $(W \wedge C) \wedge S=W \wedge S \geqslant V^{\prime} \wedge S>0 ; \quad \mathrm{b} V \wedge A \leqslant \mathrm{~b}_{A} V^{\prime}$, weil $V^{\prime} \leqslant V$ sowie $\bar{V} \wedge A \leqslant \bar{W} \wedge A \leqslant c X \wedge A=\bar{V}^{\prime} \wedge A$ und daher $\mathrm{b} V \wedge A=(\bar{V} \wedge \mathrm{c} V) \wedge A \leqslant\left(\bar{V}^{\prime} \wedge A\right) \wedge\left(\mathrm{c} V^{\prime} \wedge A\right)$ $=\mathrm{b}_{A} V^{\prime}$. Aus der letzten Beziehung folgt nach $2.1 \operatorname{dim} \mathrm{b} V \wedge A \leqslant n-1$. Das Soma $V$ besitzt also alle in der Bedingung des Satzes geforderten
Eigenschaften.

s) Vergl. auch den Hilfssatz von \$. 296.

\section{3. Summen- und Zerspaltungssätze}

Die ersten Sätze dieses Paragraphen (3.1-3.4) behandeln den Schluß ron den Dimensionen einzelner Somen auf die Dimension ihrer Vereinigung.

3.1. Voraussetzung: $A$ und $B$ seien fremde, in ihrer Vereinigung abgeschlossene Somen; $-1 \leqslant n \leqslant \infty$.

Behauptung: Aus $\operatorname{dim} A \leqslant n$ und $\operatorname{dim} B \leqslant n$ folgt $\operatorname{dim} A: B \leqslant n$.

Beweis. Es kann ohne Beschränkung der Allgemeinheit $A \vee B=E$ angenommen werden. Für $n=\infty$ ist die Behauptung trivial, ebenso für $n=-1$. Nun sei $0 \leqslant n<\infty$ und es werde ein Soma $S>0$ sowie eine beliebige Lmgebung $C$ von $S$ vorgelegt. Es sei etwa $S \wedge A>0$. Dann gibt es wegen $\operatorname{dim} A \leqslant n \mathrm{zu} \quad S \wedge A$ und der Umgebung $C \wedge A$ in $A$ von $S \wedge A$ ein in $A$ offenes Soma $V \leqslant U \therefore A$ mit $V \wedge(S \wedge A)>0$ und $\operatorname{dim} b_{A} V \leqslant n-1$. $A$ und $B$ sind offen und fremd; infolgedessen ist $F$ auch in $E$ offen und es gilt $\mathrm{b}_{4} T=\mathrm{b} r$, also $\operatorname{dim} \mathrm{b} \Gamma \leqslant n-1$. Daraus folgt $\operatorname{dim} E \leqslant n, w, z . \mathrm{b} . \mathrm{w}$.

3.2. Voraussetzung: $\mathfrak{S}$ sei collständig normal, $\mathcal{A}$ und $B$ seien beliebige Somen; $-1 \leqslant m<\infty ;-1 \leqslant n<\infty$.

Behauptung: $A$ us $\operatorname{dim} A \leqslant m$ und $\operatorname{dim} B \leqslant n$ folgt $\operatorname{dim} A \vee B \leqslant m+$ $+n+1$.

Beweis (Hurewiez-Wallman [1], S. 28, Bem. B)) (durch vollständige Induktion). Es kann ohne Beschränkung der Allgemeinheit $A \vee B=E$ angenommen werden. Für $n=m=-1$ ist die Behauptung evident. Sie sei nun für $-2 \leqslant m+n \leqslant p-1$ bereits bewiesen. Wir zeigen, daß sie auch für $m+n=p$ gilt. Es sei also $m+n=p$. Ferner seien ein beliebiges Soma $S>O$ und eine beliebige Cmgebung $U$ von $S$ gegeben. Es sei etwa $S \wedge A>0$. Dann gibt es nach 2.3 wegen $\operatorname{dim} A \leqslant m$ ein offenes Soma $V \leqslant U$ mit $T \therefore S \geqslant V \wedge(S \wedge A)>O$ und $\operatorname{dim} b V \wedge A \leqslant m-1=m^{\prime}$; dabei gilt wegen $\operatorname{dim} B \leqslant n$ nach 2.1 außerdem $\operatorname{dim} \mathrm{b} F \wedge B \leqslant n$. Weil $m^{\prime}+n=p-1$ ist, kann die Induktionsroraussetzung auf die Somen $\mathrm{b} V \wedge A$ und $\mathrm{bV} \therefore B$ angewandt werden: $\operatorname{dim} \mathrm{b} F \leqslant m^{\prime}+n+1=m+n$. Daraus folgt $\operatorname{dim} E \leqslant m+$ $+n+1$; die Behauptung gilt also auch für $m+n=p$, w. z. b. w.

HUFSSATZ. $\mathfrak{i}$ sei ein $T_{1}$-topologischer (und klassisch-topologischer) $\sigma$-Boole-Terband mit abzählbarer Basis. $\mathfrak{B}$ ist dann und nur dann höchstens 0-dimensional, wenn es zu je zwei fremden abgeschlossenen Somen $\mathcal{A}^{\prime}, A^{\prime \prime}$ zuei fremde offene Somen $G^{\prime}, G^{\prime \prime}$ mit $E=G^{\prime}, G^{\prime \prime}, A^{\prime} \leqslant G^{\prime}$ und $A^{\prime \prime} \leqslant G^{\prime \prime}$ gibt.

Beweis. (a) Dann ${ }^{6}$ ). Die Bedingung des Satzes sei erfüllt; ferner seien ein beliebiges Soma $S>0$ und eine beliebige Umgebung $U$ von $S$

6) Für diesen Teil des Beweises braucht $\Im$ nur als $T_{1}$-topologiseher (und klassischtopologischer) Boole-Yerband vorausgesetzt zu werden. 
gegeben. Da $\mathfrak{S} T_{1}$-topologisch ist, enthält $S$ ein abgeschlossenes Teilsoma $P>0 . P$ ist fremd zu dem abgeschlossenen Soma $c U$; voraussetzungsgemäß gibt es daher zwei fremde offene Somen $G^{\prime}, G^{\prime \prime}$ mit $P \leqslant G^{\prime}$, $\mathrm{c} U \leqslant G^{\prime \prime}$ und $E=G^{\prime} \vee G^{\prime \prime}$. Danm ist $G^{\prime} \leqslant U, G^{\prime} \wedge S>0$ und $\mathrm{b} G^{\prime}=O$, also $\operatorname{dim} \mathrm{b} G^{\prime}=-1$. Daraus folgt $\operatorname{dim} \mathfrak{B} \leqslant 0$.

(b) Nur dann. Es sei dim $\mathfrak{B} \leqslant 0$; ferner seien zwei beliebige fremde abgeschlossene Somen $A^{\prime}, A^{\prime \prime}$ gegeben. Dann ist die Existenz zweier fremder offener Somen $G^{\prime}, G^{\prime \prime}$ mit $A^{\prime} \leqslant G^{\prime}, A^{\prime \prime} \leqslant G^{\prime \prime}$ und $G^{\prime} \vee G^{\prime \prime}=E$ nachzuweisen. Ist $A^{\prime}=O$, so haben $G^{\prime}=O, G^{\prime \prime}=E$ die ger ünschten Eigenschaften. Nun sei $A^{\prime}>0$. Da $\operatorname{dim} \mathfrak{B} \leqslant 0$, gibt es nach 1.2 eine abzählbare Basis $\mathfrak{B}$ von $\mathfrak{B}$, deren Elemente leere Begrenzungen haben. Da die Somen $c A^{\prime}$ und $c A^{\prime \prime}$ offen sind, sind sie Vereinigungen von Somen $\epsilon \mathfrak{B}$; die in mindestens einer dieser Vereinigungen vorkommenden Somen $\epsilon \mathfrak{B}$ seien in der Folge $U_{1}, U_{2}, \ldots$ angeordnet. Für jedes $k=1,2, \ldots$ gilt dann $U_{k \wedge} A^{\prime}=O$ oder $U_{k} \wedge A^{\prime \prime}=O$ (oder beides). Von nun an schließt man weiter wie bei Hurewicz-Wallman [1], s. 16 (mit $A^{\prime}=C, A^{\prime \prime}=K, G^{\prime}=C^{\prime}$ and $G^{\prime \prime}=K^{\prime}$ ).

3.3. Voraussetzung: $\mathfrak{B}$ sei ein $\mathcal{S}$-Verband; in $\mathfrak{B}$ seien abzählbar viele in ihrer Vereinigung abgeschlossene Somen $F_{i}$ gegeben $;-1 \leqslant n \leqslant \infty$.

Behauptung: Aus $\operatorname{dim} F_{i} \leqslant n$ für $i=1,2, \ldots$ folgt $\operatorname{dim} \bigvee_{i=1}^{\infty} F_{i} \leqslant n$.

Beweis. Für $n=\infty$ ist die Behauptung sicher richtig, ebenso für $n=-1$; für $0 \leqslant n<\infty$ soll sie durch vollständige Induktion bewiesen werden.

Es sei zunächst $n=0$. Ohne Beschränkung der Allgemeinheit kann $\bigvee_{i=1}^{\infty} F_{i}=E$ (und jedes $F_{i}$ als abgeschlossen) angenommen werden. Dann verläuft der Beweis analog wie bei Hurewicz-Wallman [1], S. 18-19.

Es werde nun vorausgesetzt, daß 3.3 für $0 \leqslant n<m-1$ bereits bewiesen ist. Dann gilt für einen $S$-Verband $\mathfrak{B}$ und eine ganze Zahl $k$ mit $0 \leqslant k \leqslant m$ die folgende $Z$ wischenbemerkung: (*) Ist $\operatorname{dim} \mathfrak{B} \leqslant k$, so gibt es zwei fremde Somen $N^{\prime}, N^{\prime \prime}$ mit $E=N^{\prime} \vee N^{\prime \prime}$,
$\operatorname{dim} N^{\prime} \leqslant k-1$ und $\operatorname{dim} N^{\prime \prime} \leqslant 0$.

Da nämlich $\mathfrak{B}$ als $S$-Terband eine abzählbare Basis besitzt, folgt aus 1.2 die Existenz einer abzählbaren Basis $\left\{B_{i}\right\}_{i=1,2, \ldots}$ von $\mathfrak{Y}$ mit $\operatorname{dim} \mathrm{b} B_{i} \leqslant k-1 \leqslant n-1$ für jedes $i=1,2, \ldots$ Nun sei $N^{\prime}=\bigvee_{i=1}^{\infty} \mathrm{b} B_{i} . \quad\left(N^{\prime}\right.$ existiert, weil $\mathfrak{B}$ ein $\sigma$-Verband ist.) Da jedes $\mathrm{b} B_{i}$ abgeschlossen ist, folgt auf Grund der Induktionsroraussetzung aus 3.3 dim $N^{\prime} \leqslant k-1$. Das Komplement $N^{\prime \prime}$ ron $N^{\prime}$ sehließlich ist höchstens 0 -dimensional.
Tun sei $\operatorname{dim} F_{i} \leqslant m$ für $i=1,2, \ldots$ Ohne Beschränkung der Allgemeinheit kann $\bigvee_{i=1}^{\infty} F_{i}=E$ (und jedes $F_{i}$ als abgeschlossen) angenommen werden. Dann beweist man $\operatorname{dim} E \leqslant m$ wie bei Hurewicz-Wallman [1], S. 31-32 unter Berücksichtigung der Tatsache, daß in einem $S$-Verband jedes offene Soma ein $F_{\sigma}$ (und jedes abgeschlossene Soma ein $G_{\delta}$ ) ist (Sikorski [4], S. 185). Mit dim $E \leqslant m$ ist 3.3 rollständig bewiesen und gleichzeitig. die Gültigkeit der Zwischenbemerkung (*) für jede endliche ganze Zahl $k \geqslant 0$ sichergestellt.

3.4. Voraussetzung: $\mathfrak{B}$ sei ein S-Terband, $A$ ein beliebiges Soma, $B$ ein Soma, das zugleich $F_{\sigma}$ und $G_{\delta}$ in $A \vee B$ ist $\left.{ }^{7}\right) ;-1 \leqslant n \leqslant \infty$.

Behauptung: Aus $\operatorname{dim} A \leqslant n$ und $\operatorname{dim} B \leqslant n$ folgt $\operatorname{dim} A \vee B \leqslant n$.

Beweis wie bei Menger [2], S. 114-115.

Die zum Bewreis des Summensatzes 3.3 herangezogene und bewiesene Zwischenbemerkung betrifft die Zerspaltung eines Somas in Somen niedrigerer Dimension. In den Sätzen 3.5 bis 3.7 sind solche Zerspaltungstatsachen aufgeführt.

3.5. Voraussetzung: $\mathfrak{B}$ sei ein $S$-Verband; $0 \leqslant n<\infty$.

Behauptung: Ist dim $\mathfrak{B} \leqslant n$, so gibt es zwei fremde Somen $N^{\prime}, N^{\prime r}$ mit $E=N^{\prime} \vee N^{\prime \prime}, \operatorname{dim} N^{\prime} \leqslant n-1$ und $\operatorname{dim} N^{\prime \prime} \leqslant 0$.

Beweis: Satz 3.3, Zwischenbemerkung $(*)$.

3.6. Voraussetzung: $\mathfrak{B}$ sei vollständig normal; $0 \leqslant n<\infty$.

Behauptung: Damit $\operatorname{dim} \mathfrak{B} \leqslant n$ sei, ist hinreichend und, falls $\mathfrak{B}$ ein S-Ferband ist, auch notucendig, daß es $n+1$ paarweise fremde, höchstens 0 -dimensionale Somen $A_{i}$ mit $E=V_{i=1}^{n+1} A_{i}$ gibt.

Beweis: 3.2 und 3.5 .

3.7. Voraussetzung: 3 sei ein S-Terband; $-1 \leqslant n<\infty$.

Behauptung: Ist $\operatorname{dim} \mathfrak{B}=n$, so gibt es zu je zwei ganzen Zahlen $p, q$ mit $p \geqslant q \geqslant-1$ und $p+q=n-1$ zwei fremde Somen $P, Q$ mit $E=P \vee Q$, $\operatorname{dim} P=p$ und $\operatorname{dim} Q=q$.

Betreis: 3.6.

Der Zerspaltungssatz 3.5 ermöglicht es, den Hilfssatz von Seite 293 zu verallgemeinern $\left.{ }^{8}\right)$ :

7) Diese Voraussetzung ist nach einer eben gemachten Bemerkung (vergl. Sikorski [4], S. 185), z. B. dann erfüllt, wenn $B$ in $A \vee B$ abgeschlossen (oder offen) ist. 8) Dieser Satz 3.8 betrifft eine Eigenschaft von $\mathfrak{V}$, die in $\S 6$ systematisch behandelt werden soll. Wir beweisen ihn schon jetzt, weil aus ihm (für $S$-Verbände) die

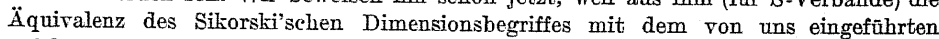
gefolgert werden kann und es wünschenswert erscheint, diese Äquivalenz möglichst. frühzeitig festzustellen. 
3.8. Voraussetzung: $\mathfrak{B}$ sei ein. $T_{1}-$ Verband, $A$ ein beliebiges Soma; $0 \leqslant n<\infty$.

Behauptung: Damit $\operatorname{dim} A \leqslant n$ sei, ist hinreichend und, falls $\mathfrak{B}$ ein S-Ferband ist, auch notwendig, daß es zu je zwei fremden abgeschlossenen Somen $A^{\prime}, A^{\prime \prime}$ ein abgeschlossenes Soma $B$ und zwei fremde offene Somen $G^{\prime}, G^{\prime \prime}$ mit $\mathrm{c} B=G^{\prime} \vee G^{\prime \prime}, A^{\prime} \leqslant G^{\prime}, A^{\prime \prime} \leqslant G^{\prime \prime}$ und $\operatorname{dim} B \wedge A \leqslant n-1$ gibt.

Beweis. (a) Hinreichend. Es sei die Bedingung des Satzes erfüllt; ferner seien ein nicht-leeres Soma $S \leqslant A$ sowie eine beliebige Umgebung $U$ von $S$ vorgelegt. Da $\mathfrak{B} T_{1}$-topologisch ist, existiert ein abgeschlossenes nicht-leeres Soma $P \leqslant S$. Die Somen $P$ und $\mathrm{c} U$ sind abgeschlossen und fremd; nach Voraussetzung gibt es daher ein abgeschlossenes Soma $B$ und zwei fremde offene Somen $G^{\prime}, G^{\prime \prime}$ mit $\mathrm{c} B=G^{\prime} \vee G^{\prime \prime}, P \leqslant G^{\prime}, c U \leqslant G^{\prime \prime}$ und $\operatorname{dim} B \wedge A \leqslant n-1$. Dann ist $G^{\prime} \leqslant U, \quad G^{\prime} \wedge S>0$ und $b G^{\prime} \leqslant B$, also $\operatorname{dim} \mathrm{b} G^{\prime} \wedge A \leqslant n-1$. Nach 2.3 folgt hieraus $\operatorname{dim} A \leqslant n$.

(b) Notwendig. $\mathfrak{B}$ sei ein $S$-Verband. Für $\operatorname{dim} A \leqslant 0$ verläuft der Beweis wie bei Hurewicz-Wallman [1], S. 16-18, Bem. F), für $\operatorname{dim} A \leqslant n$ ( $n$ beliebig $\geqslant 0$ ) wie bei Hur ewicz-Wallman [1], S. 34, Bem. B).

Aus dem eben bewiesenen Satz folgt unmittelbar:

3.9. Für S-Verbände ist der Sikorski'sche Dimensionsbegriff mit dem. auf Seite 290 definierten äquivalent.

Als weitere Anwendung von 3.8 sei zum Schluß noch ein später benötigter Hilfssatz angegeben, der eine Verallgemeinerung des Satzes 2.3 darstellt.

HMFSSATZ. A sei ein beliebiges Soma; $0 \leqslant n<\infty$. Damit $\operatorname{dim} A \leqslant n$ sei, ist hinreichend und, falls $\mathfrak{B}$ ein $\mathcal{S}-\nabla$ erband ist, auch notwendig, daß es zu jedem Soma $S>0$ und jeder Umgebung $U$ von $S$ ein offenes Soma $V \leqslant U$ mit $V \wedge S>O$ und $\operatorname{dim} \mathrm{b} \nabla \wedge A \leqslant n-1$ gibt.

Beweis. (a) Hinreichend. Mit der Bedingung des Satzes ist erst recht die Bedingung von 2.3 erfüllt; daraus folgt $\operatorname{dim} A \leqslant n$.

(b) Notwendig. Es sei $\mathfrak{B}$ ein $S$-Verband und $\operatorname{dim} A \leqslant n$; ferner seien ein nicht-leeres Soma $S$ und eine beliebige Umgebung $U$ von $S$ vorgelegt. $\mathrm{Da} \mathfrak{B}$ als $\mathcal{S}$-Verband $T_{1}$-topologisch ist, gibt es ein nicht-leeres abgeschlossenes Soma $P \leqslant S$. Die Somen $P$ und $\mathrm{c} U$ sind abgeschlossen und fremd; nach 3.8 gibt es daher ein abgeschlossenes soma $B$ und zwei offene, fremde Somen $G^{\prime}, G^{\prime \prime}$ mit $\mathrm{c} B=G^{\prime} \vee G^{\prime \prime}, P \leqslant G^{\prime}, \mathrm{c} U \leqslant G^{\prime \prime}$ und $\operatorname{dim} B \wedge A \leqslant n-1$. Setzt man dann $G^{\prime}=V$, so ist $V \leqslant U, V \wedge S>O$ und $\mathrm{b} \Gamma \leqslant B$, also $\operatorname{dim} \mathrm{b} V: A \leqslant n-1$. Das Soma $V$ leistet somit das Verlangte.

\section{4. Die dimensionelle Struktur von $s$-Verbänden}

In diesem Paragraphen liege durchwegs ein $S$-Verband $\& 3$ mit $E \neq O$ vor.

Wegen des Fehlens ron Punkten gestaltet sich die Definition des dimensionellen Verhaltens ron $\mathfrak{B}$ in seinen Somen etwas komplizierter als bei einem Raum; die Ergebnisse der Strukturuntersuchungen sind jedoch dieselben.

Definition. $A$ sei ein beliebiges nicht-leeres Soma, $k$ eine ganze Zahl $\geqslant 0$.

(a) $\mathfrak{B}$ heiße höchstens $k$-dimensional in $A$, wenn es zu jedem nicht-leeren Teilsoma $S$ von $A$ und jeder Umgebung $U$ ron $S$ ein offenes Soma $V \leqslant C$ mit $F \wedge S>0$ und $\operatorname{dim} \mathrm{b} T \leqslant k-1$ gibt.

(b) $\mathfrak{B}$ heiße mindestens $k$-dimensional in $A$, wenn $\mathbb{B}$ in keinem nicht-leeren Teilsoma ron $A$ höchstens $(k-1)$-dimensional ist.

(c) $\mathbb{B}$ heiße (genan) $k$-dimensional in $\mathcal{A}$, wenn $\mathfrak{B}$ in $A$ sowohl höchstens als auch mindestens $k$-dimensional ist.

Ersichtlich ist dim $\mathbb{Q} \leqslant k$, wenn und nur wenn $\mathfrak{B}$ in $E$ höchstens $k$-dimensional ist. Aus der Definition ergibt sich ferner unmittelbar:

4.1. Voraussetzung: A sei ein beliebiges nicht-leeres soma; $0 \leqslant k<\infty$.

Behauptung: (a) Ist $\mathfrak{B}$ höchstens $k$-dimensional in $A$, so auch in jedem nicht-leeren Teilsoma ron A.

(b) Ist $\mathfrak{B}$ mindestens $k$-dimensional in $A$, so auch in jedem nicht-leeren Teilsoma von A.

Ist $\mathcal{A}$ ein nicht-leeres Soma, so braucht $\mathfrak{B}$ in $\mathcal{A}$ weder höchstens $(k-1)$-dimensional noch mindestens $k$-dimensional zu sein. In der Tat: Gibt es in $\mathfrak{B}$ zwei (fremde) nicht-leere Somen $B$ und $C$ derart, daß $\mathfrak{B}$ in $B$ höchstens $(k-1)$-dimensional und in $C$ mindestens $k$-dimensional ist, so ist $\mathfrak{B}$ in $A=B \cup C$ weder höchstens $(k-1)$ - noch mindestens $\mathrm{k}$-dimensional. Bezeichnet man also mit $D^{k}$ das Srstem aller nicht-leeren Somen von $\mathfrak{B}$, in denen $\mathfrak{B}$ mindestens $k$-dimensional, und mit $\mathfrak{I}^{k-1}$ das $\mathbf{S y s t e m}$ aller nicht-leeren Somen ron $\mathfrak{B}$, in denen $\mathfrak{B}$ höchstens $(k-1)$-dimensional ist, so enthalten (für ein festes $k$ ) die Systeme $\mathfrak{D}^{k}$ und $\mathfrak{T}^{k-1}$ zusammen im allgemeinen nicht alle nicht-leeren Somen von $\mathfrak{B}$; in einem gemissen Sinn liefern sie aber, wie aus 4.2 hervorgehen wird, trotzdem eine rollständige Klassifizierung der Somen ron $\mathfrak{B}$.

Ist $\mathfrak{B}$ in einem nicht-leeren Soma $A$ mindenstens $k$-dimensional, so ist $\mathfrak{B}$ in $A$ erst recht mindestens $(k-1)$-dimensional. In der obigen Bezeichnungsweise heißt dies $\mathfrak{D}^{k} C \mathfrak{D}^{k-1}$. Da $\mathfrak{B}$ in jedem nicht-leeren Soma mindestens 0-dimensional ist, so gilt ferner, weun man zu $\mathfrak{D}^{\circ}$ noch das Null- 
soma hinzurechnet, $D^{0}=\mathfrak{B}$. Weitere Untersuchungen der Systeme $\mathfrak{D}^{k}$ und $\mathfrak{T}^{k-1}$ führen auf den folgenden Satz 4.2 .

HUfFSATZ 1. Zu jeder abzählbaren Basis $\mathfrak{B}$ von $\mathfrak{B}$ gibt es eine Doppelfolge $\left\{B_{i}^{k}\right\}\left(B_{i}^{k} \in \mathfrak{B}\right.$ fïr $\left.i, k=1,2, \ldots\right)$ mit folgenden Eigenschaften:

(1) Für jede natürliche Zahl k ist $\bigvee_{i=1}^{\infty} B_{i}^{k}=E$.

(2) Zu je zuei Somen $G, H \in \mathfrak{B}$ mit $\bar{G} \leqslant H$ gibt es eine natürtiche Zah $k=k(G ; H)$ derart, daß für kein $i=1,2, \ldots$ sowohl $B_{i}^{k} \wedge G>0$ als auch $B_{i}^{k} \wedge \mathrm{c} H>0$ gilt, $\left.{ }^{9}\right)$.

Beweis. Da $\mathfrak{B}$ abzählbar ist, gibt es höchstens abzählbar viele Paare $(G, H)$ von Somen aus $\mathfrak{B}$ mit $\bar{G} \leqslant H$. (Ein solches Paar existiert sicher, da $G=O$ gesetzt werden kann.) Diese Paare seien in einer Folge $\left\{\left(G^{k}, H^{k}\right)\right\}$, $k=1,2, \ldots$ angeordnet (falls es nur endlich viele, etwa $n$, solcher Paare gibt, sei $G^{k}=G^{n}$ und $H^{k}=H^{n}$ für $\left.k>n\right)$. Für jedes $k$ sind die Somen $H^{k}$ und $c \overline{G^{k}}$ offen und es g lt $H^{k} \vee c \overline{G^{k}}=E$; als offene Somen sind $H^{k}$ und $c \overline{G^{k}}$ Vereinigurg $n$ von Basiselementen. Man ordnet nun für jede natürliche Zahl $k$ die in diesen beiden Vereinigungen auftretenden (abzählbar vielen) Somen $\epsilon \mathfrak{B}$ in einer Folge $B_{1}^{k}, B_{2}^{k}, \ldots$ an (falls es nur endlich viele, etwa $m$ solcher Somen gibt, sei $B_{i}^{k}=B_{m}^{k}$ für $\left.i>m\right)$. Die Doppelfolge $\left\{B_{i}^{k}\right\}$ leistet dann das Verlangte.

HMFSSATZ 2. Es sei $\mathfrak{M}$ ein System offener Somen und $\mathfrak{A}$ das System aller Somen $A$, für die es zu jedem nicht-leeren Teilsoma $S \leqslant A$ und jeder Umgebung $U$ von $\mathbb{S}$ ein Soma $W \in \mathfrak{B}$ mit $W \leqslant U$ und $W \wedge S>0$ gibt. Dann existiert das Soma $D=\bigvee_{\mathfrak{X}} A$, liegt in $\mathfrak{U}$ und ist ein $\left.G_{\delta}{ }^{19}\right)$.

Beweis. Es sei $\left\{B_{i}^{k}\right\}$ eine gemäß Hilfssatz 1 konstruierte Doppelfolge; für jede natürliche Zahl $k$ sei weiter $W^{k}$ die Vereinigung aller $W \in \mathfrak{B}$, die für irgend einen Index $i$ im Soma $B_{i}^{k}$ enthalten sind. Als Vereinigung offener Somen ist jedes $W^{k}$ offen; das Soma $D=\bigwedge_{k=1}^{\infty} W^{k}$ ist also ein $G_{\delta}$. Wir behaupten, daß dieses Soma $D$ die Vereinigung $\underset{\mathfrak{r}}{\vee} A$. ist. Erstens ist jedes $A \in \mathfrak{U}$ in $D$ enthalten. Angenommen nämlich, ein Soma $A \in \mathfrak{U}$ wäre nicht in $W^{k}$ enthalten. Dann ist $S=A \wedge \mathrm{c} W^{k}>0$ und es gibt wegen (1) von Hilfssatz 1 einen Index $i$ mit $S_{\wedge} B_{i}^{k}>0$. Nun ist $S_{\wedge} B_{i}^{k}$ ein nicht-leeres Teilsoma von $A$ und $B_{i}^{k}$ eine Umgebung von $S_{\wedge} B_{i}^{k}$; infolgedessen gibt es ein Soma $W \in \mathfrak{W}$ mit $W \leqslant B_{i}^{k}$ und $W \wedge S \wedge B_{i}^{k}>0$. Aus $W \leqslant B_{i}^{k}$ folgt aber $W \leqslant W^{k}$ und hieraus wegen $W \wedge S \wedge B_{i}^{k}>0$ weiter $W^{k} \wedge S>0$, was im Widerspruch zu $S=A \wedge \subset W^{k}$ steht. Zweitens ist $D \in \mathfrak{A}$. Zum Beweis dieser Be-

") Menger [2], S. 60, "Theorem von den ausgezeichneten Doppelfolgen".

10) Menger [2], S. 68, "Theorem über Gleichwertigkeitsteile". hauptung betrachten wir irgend ein nichtleeres Teilsoma $S$ von $D$ und eine beliebige Umgebung $U$ von $S$. Es existieren nun wegen der Regularität ron $\mathfrak{B}$ in $\mathfrak{B}$ zwei Somen $G, H$ mit $G \wedge S>O, \bar{G} \leqslant H$ und $H \leqslant U$, ferner die Zahl $k=k(G ; H)$ im Sinne von (2) des Hilfssatzes 1 und schließlich wegen $0<G \wedge S \leqslant S \leqslant D \leqslant W^{k}$ ein $B_{i}^{k}$ und ein $W \leqslant B_{i}^{k}$ mit $G \wedge S \wedge W>0$. Dann ist $G \wedge B_{i}^{k}>0$, also $B_{i}^{k} \leqslant H \leqslant U$ nach (2) ron Hilfssatz 1 , also $W \leqslant U$; außerdem ist $S \wedge W>O$. Infolgedessen ist $D \in \mathfrak{A}$ nach Definition ron $\mathfrak{A}$. Aus $A \leqslant D$ für jedes $A \in \mathfrak{A}$ und $D \in \mathfrak{I}$ folgt schlieBlich $D=\underset{\mathfrak{r}}{ } A$.

4.2. Für jedes $k=0,1,2, \ldots$ existieren zuei Somen $D^{k}$ und $T^{k-1}$ mit folgenden Eigenschaften:

(1) $D^{k_{\vee}} T^{k-1}=E ; D^{k} \wedge T^{k-1}=0$;

(2) $\mathfrak{B}$ ist dann und nur dann in $A$ mindestens $k$-dimensional, wenn $O<A \leqslant D^{k}$ ist

(3) $\mathfrak{B}$ ist für $k \geqslant 1$ dann und nur damn in A höchstens $(k-1)$-dimensional, wenn $O<A \leqslant T^{k-1}$ ist; $T^{-1}=0$;

(4) $D^{k}$ ist ein $F_{\sigma}, T^{k-1}$ ist ein $G_{\delta}^{11}$ ).

Bew eis (Menger [2], S. 127). Es seien $\mathfrak{D}^{k}$ und $\widetilde{I}^{k-1}$ die auf Seite 297 definierten Somensysteme. Wegen $\mathfrak{D}^{0}=\mathfrak{B}$ leisten für $k=0$ die Somen $D^{0}=E$ und $T^{-1}=0$ das Verlangte. Nun sei $k \geqslant 1$. $\mathfrak{I}^{k-1}$ ist die Menge aller nicht-leeren Somen $A$, für die es zu jedem nicht-leeren Teilsoma $S$ von $A$ und jeder Umgebung $U$ von $S$ ein offenes Soma $V \leqslant U$ mit $V \wedge S>0$ und $\operatorname{dim} \mathrm{b} V \leqslant k-2$ gibt. Bezeichnet man daher mit $\mathfrak{M}$ das System aller offenen Somen $V$ mit $\operatorname{dim} \mathrm{b} V \leqslant k-2$, so folgt aus dem Hilfssatz 2, daß $T^{k-1}=\bigvee A$ existiert, in $\mathfrak{I}^{k-1}$ liegt und ein $G_{\delta}$ ist. Es existiert dann $\mathfrak{T}^{k-1}$

anch das Soma $D^{k}=\mathrm{e} T^{k-1}$ und ist ein $F_{\sigma}$. Daß (3) gilt, folgt aus der Definition ron $\mathfrak{I}^{k-1}$ und aus 4.1. Hieraus folgt weiter (2).

KOROLLAR. Es gilt $E=D^{0} \geqslant D^{1} \geqslant \ldots \geqslant D^{k-1} \geqslant D^{k} \geqslant \ldots$

Der eben bewiesene Satz ermöglicht folgende

Definition. Das durch 4.2 erklärte Soma $D^{k}$ heiße der $k$-te Dimensionsteil von $\mathfrak{D}$.

Die Dimension ron $\mathfrak{B}$ steht mit seinen Dimensionsteilen in folgendem Zusammenhang:

4.3. Voraussetzung: $0 \leqslant n<\infty$.

Behauptung: Es ist $\operatorname{dim} \mathfrak{B} \leqslant n$, wenn und nur wenn $D^{n-1}=0$ ist.

Beweis. Es sei $D^{n+1}=O$. Nach 4.2 ist dann $T^{n}=E$, also $\mathfrak{B}$ in $E$ höchstens $n$-dimensional. Definitionsgemäß bedeutet dies $\operatorname{dim} \mathfrak{B} \leqslant n$. Die Umkehrung dieser Schlüsse zeigt, $\operatorname{da} \beta$ aus $\operatorname{dim} \mathfrak{B} \leqslant n$ folgt $D^{n+1}=0$.

11) Die Somen $D^{k}$ und $T^{k-1}$ sind durch (2) nnd (3) eindeutig bestimmt. 
Die nächsten Sätze betreffen die Dimension der Dimensionsteile und ihrer Komplemente.

4.4. Voraussetzung: $0 \leqslant k<\infty$.

Behauptung: Es ist $\operatorname{dim} T^{k-1} \leqslant k-1$.

Beweis. Für $k=0$ ist die Behauptung evident. Nun sei $k \geqslant 1$. Nach 4.2 (3) ist $\mathfrak{B}$ in $T^{k-1}$ höchstens $(k-1)$-dimensional. Also gibt es zu jedem nicht-leeren Teilsoma $S$ von $T^{k-1}$ und jeder Umgebung $U$ von $S$ ein offenes Soma $T \leqslant U$ mit $V \wedge S>O$ und $\operatorname{dim} \mathrm{b} V \leqslant k-2$. Nun ist $\mathrm{b} V \wedge T^{k-1} \leqslant \mathrm{~b} V$, also $\operatorname{dim} \mathrm{b} \Gamma \wedge T^{k-1} \leqslant k-2$. Aus 2.3 folgt jetzt die Behauptung.

4.5. Toraussetzung: $0 \leqslant k<\infty ; D^{k}>0$.

Behauptung: Es ist dim $D^{k} \geqslant k-1$.

Statt 4.5 beweisen wir den folgenden, schärferen Satz:

4.5a. Voraussetzung: $0 \leqslant k<\infty ; D^{k}>0$.

Behauptung: Für jedes nicht-leere, in $D^{k}$ offene Soma $T^{k}$ ist $\operatorname{dim} C^{k} \geqslant k-1$.

Beweis (Menger [2], S. 135-137, "Zweites Fundamentaltheorem"). Für $k=0$ ist die Behauptung trivial, weil jedes Soma mindestens $(-1)$ -dimensional ist. Nun sei $k \geqslant 1$ und es sei ein nicht-leeres, in $D^{k}$ offenes Soma $C^{k}$ vorgelegt. Es gibt dann ein (in $\mathfrak{B}$ ) offenes Soma $U_{0}$ mit $U^{k}$ $=U_{0} \wedge D^{k}$. Nach 4.2 ist nun $\mathfrak{B}$ in $U_{0} \wedge c D^{k}$ (falls dieses Soma nicht leer ist) höchstens ( $k-1)$-dimensional. Hieraus und aus der Regularität von $\mathfrak{B}$. folgt, daß es zu jedem nicht-leeren Teilsoma $P$ von $U_{0} \wedge c D^{k}$ und jeder Umgebung $C$ von $P$ ein offenes Soma $W$ mit $W \wedge P>O, \bar{W} \leqslant U_{0} \wedge U$ und $\operatorname{dim} \mathrm{b} W \leqslant k-2$ gibt. Bezeichnet man nun mit $\mathfrak{B}$ das System dieser Somen $W$ für jedes nicht-leere Teilsoma $P$ von $U_{0} \wedge c D^{k}$ und jede Umgebung $C$ von $P$, so enthält $\mathfrak{W}$ (weil $\mathfrak{B}$ eine abzählbare Basis besitzt) ein abzählbares Teilsystem $\mathfrak{W}_{0}=\left\{W_{1}, W_{2}, \ldots\right\}$ derart, daß es zu jedem nicht-leeren Teilsoma $P$ von $U_{0} \wedge \mathrm{c} D^{k}$ und jeder Umgebung $U$ von $P$ ein $W_{i}$ mit $W_{i} \wedge P>0, \bar{W}_{i} \leqslant U_{0} \wedge U$ und $\operatorname{dim} \mathrm{b} W_{i} \leqslant k-2$ gibt (Beweis analog wie für 1.2). Setzt man dann $\bigvee_{i=1}^{\infty} \mathrm{b} W_{i}=B$, so ist $\operatorname{dim} B \leqslant k-2$ nach 3.3 und $B \leqslant C_{0}$; weiter gilt, wenn $U_{0}: \mathrm{c} D^{k} \wedge \mathrm{c} B=A$ gesetzt wird, $\operatorname{dim} A \leqslant 0$. Wendet man nun den Hilfssatz von Seite 296 auf das Soma $A$ an, so erhält man mit Berücksichtigung der Regularität von $\mathfrak{B}$ zu jedem nicht-leeren Soma $S \in \mathfrak{B}$, also erst recht zu jedem nicht-leeren Soma $P \leqslant U_{0}$ und zu jeder Umgebung $U$ von $P$ ein offenes Soma $V$ mit $\bar{V} \leqslant U_{0} \wedge U, \nabla \wedge P>O$ and $\operatorname{dim} \mathrm{b} V \wedge A=-1$. Für jedes solche $V$ ist $\mathrm{b} V \leqslant U_{0}$ und $\mathrm{b} V \wedge A=0$, also $\mathrm{b} V \leqslant\left(C_{0} \wedge D^{k}\right) \vee B$. - Wäre nun im Gegensatz zur Behauptung $\operatorname{dim} C_{0} \therefore D^{k} \leqslant k-2$, so auch $\operatorname{dim}\left(C_{0} \wedge D^{k}\right) \vee B \leqslant k-2 . D^{k}$ ist nämlich nach 4.2 ein $F_{\sigma}, C_{0} \wedge D^{k}$ also ein $F_{\sigma}$ in $U_{0}$ und (wegen $\left(U_{0} \wedge D^{k}\right) \vee B \leqslant U_{0}$ ) auch ein $F_{\sigma}$ in $\left(C_{0} D^{k}\right) B ; B$ ist definitionsgemä $\beta$ ein $F_{\sigma}$, also anch ein $F_{\sigma}$ in
$\left(C_{0}: D^{k}\right) B$; infolgendessen ist $\left(C_{0} \wedge D^{k}\right) \vee B$ Vereinigung abzählbar vieler, in $\left(C_{0}: D^{k}\right): B$ abgeschlossener Somen, die auf Grund der Annahme $\operatorname{dim} C_{0} \therefore D^{k} \leqslant k-2$ und der Beziehung dim $B \leqslant k-2$ sämtlich höchstens. $(k-2)$-dimensional wären, so daß sich nach 3.3 in $\operatorname{der} T$ Tat $\operatorname{dim}\left(U_{0} \cdot D^{k}\right) \vee B$ $\leqslant k-2$ ergäbe. Insbesondere wäre dann auch nach $2.1 \operatorname{dim} \mathrm{b} V \leqslant k-2$ für jedes der oben bestimmten Somen $V$. Das hieße aber, daß $\mathfrak{B}$ in $\tau_{0}$ höchstens $(k-1)$-dimensional wäre, und dies bedeutete $U_{0} D^{k}=0$ nach $4.2 \mathrm{im}$ Widerspruch zur Toraussetzung $U^{k}>O$. Die Annahme $\operatorname{dim} C_{0}: D^{k} \leqslant k-2$ ist demnach falsch, es ist vielmehr $\operatorname{dim} C_{\mathbf{0}} \therefore D^{k} \geqslant k-1$.

4.6. Voraussetzung: $\mathfrak{B}$ sei kompaht; $0 \leqslant k<\infty ; D^{k}>0$.

Behauptung: $E s$ ist $\operatorname{dim} D^{k} \geqslant k$.

Beweis. Auf Grund der Voraussetzung ist $\mathfrak{B}$ atomar (Nöbeling [3]. Satz 12.2 und 12.5). Es sei nun $E^{\prime}$ die Menge aller Atome von $\mathfrak{B}$ und $\mathfrak{E}^{\prime}$ der Mengenrerband aller Teilmengen von $E^{\prime}$. Für jedes Soma $A \in \mathfrak{B}$ sei $A^{\prime}=\Phi A$ die Menge aller Atome $P \leqslant A$. Dann ist $\Phi$ ein Isomorphismus von $\mathfrak{B}$ auf einen Tnterrerband $\mathfrak{B}^{\prime}$ von $\mathfrak{E}^{\prime}$ (derart, daß für jede Somenfamilie $\left(A_{i}\right)_{i \in \mathfrak{S}}$ aus $\mathfrak{B}$, für welche $\vee A_{i}$ bzw. $\wedge A_{i}$ existiert, $\Phi \vee A_{i}=\cup \Phi A_{i}$ bzw. $\Phi \wedge A_{i}=\cap \Phi A_{i}$ ist und daß für jedes Soma $A \in \mathfrak{B} \Phi \mathrm{c} A=E^{\prime}-\Phi A$ gilt). Das Sxstem aller Mengen $\Phi B_{i}$, wobei $B_{i}$ eine abzählbare Basis von $\mathfrak{B}$ durchläuft, sei eine Basis ron $\mathscr{E}^{\prime}$. Hierdurch wird $\mathfrak{E}^{\prime}$ zu einem klassisch-topologischen Raum, und zwar zu einem kompakten, regulären $T_{1}$-Raum. mit abzählbarer Basis. $\Phi$ ist eine Homöomorphie von $\mathfrak{B}$ auf $\mathfrak{B}^{\prime}$; die $\Phi$-Bilder der offenen (abgeschlossenen) Somen von $\mathfrak{B}$ sind die offenen (abgeschlossenen) Mengen ron $\mathfrak{E}^{\prime}$.

Beachtet man nun noch, daß im atomaren Fall die Begriffe „höchstens $n$-dimensional" (Seite 290) und "höchstens $k$-dimensional in $A$ " (Seite 297) sich nicht ändern, wemn man 1. c. für $S$ nur Atome zuläßt, so ergibt sich erstens, daß für ein Soma $A \in \mathfrak{B}$ stets gilt $\operatorname{dim} A=\operatorname{dim} \Phi A$ (letztere Dimension in $\mathfrak{E}^{\prime}$ (nicht in $\mathfrak{B}^{\prime}$ ) genommen), zweitens, daß $\mathfrak{B}$ in einem Atom $P$ dann und nur dann höchstens $k$-dimensional ist, wenn $\mathfrak{E}^{\prime}$ in $\Phi P$ höchstens $k$-dimensional ist, und drittens, daß $D^{\prime k}=\Phi D^{k}$ der $k$-te Dimensionsteil ron $\mathcal{E}^{\prime}$ ist.

Num ist nach Menger [2] (Seite 128) $\operatorname{dim} D^{\prime k} \geqslant k$. Wegen $\operatorname{dim} D^{k}$ $=\operatorname{dim} D^{\prime k}$ ist also $\operatorname{dim} D^{k} \geqslant k$.

\section{§ 5. Überdeckungssätze}

In diesem Paragraphen liege wieder ein $S$-Ferband $\mathfrak{B}$ vor.

Definition. A sei ein beliebiges Soma.

(a) Ein System $\mathfrak{U}$ offener (bzw. abgeschlossener) Somen heiße eine offene (bzw. abgeschlossene) Überdeckung von $A$, wenn $A$ kein zu allen $U \in \mathfrak{U}$ tremiles Soma $>0$ enthält. 
(b) Eine Überdeckung $\mathfrak{U}$ heiße endlich, wenn $\mathfrak{U}$ nur endlich viele Elemente $U$ enthält.

(c) Eine endliche, offene (bzw. abgeschlossene) Überdeckung $\mathfrak{U}$ $=\left\{U_{1}, \ldots, U_{s}\right\}$ heiße vom Grade $\leqslant n$, wenn entweder $s \leqslant n+1$ ist oder je $n+2$ verschiedene Somen $U_{i}$ einen leeren Durchschnitt haben.

(d) Sind $\mathbb{S}$ und $\mathfrak{I}$ zwei beliebige Systeme irgend welcher Somen ron $\mathfrak{N}$, so heiße $\mathfrak{I} \mathfrak{S}$-fein oder eine Verfeinerung von $\mathfrak{S}$, wenn jedes Soma $\epsilon \mathfrak{I}$ in einem Soma $\epsilon \mathbb{S}$ enthalten ist.

Die Beziehungen zwischen dem Grad "beliebig feiner" U̇berdeckungen und der Dimension von $E$ sind Gegenstand des Satzes 5.1. Im Hinblick auf den ersten Hilfssatz und die Tatsache, daß $E$ abgeschlossen ist, bleibt es dabei gleichgültig, ob offene oder abgeschlossene Überdekkungen betrachtet werden. Beschränkt man sich auf die Eigenschaften beliebig feiner abgeschlossener Überdeckungen, so läßt sich die Aussage des Satzes 5.1 in einer gewissen Weise verallgemeinern (5.2).

Hnfssatz 1. Es sei A ein beliebiges Soma. Damit es zu einer endlichen, offenen Überdeckung $\mathfrak{U}$ von $A$ eine endliche, $\mathfrak{U}$-feine, offene Überdeckung $\mathfrak{M}$ des Somas $A$ vom Grade $\leqslant n$ gibt, ist hinreichend und, falls $A$ abgeschlossen ist, auch notwendig, daß eine endliche, $\mathfrak{U}$-feine, abgeschlossene Überdeckung 3 des Somas A vom Grade $\leqslant n$ existiert ${ }^{12}$ ).

Beweis. (a) Hinreichend. Ist $3=\left\{Z_{1}, \ldots, Z_{t}\right\}$ eine endliche, $\mathfrak{U}$ -feine, abgeschlossene Überdeckung des Somas $A$ vom Grade $\leqslant n$, so genügt es, ein endliches $\mathfrak{U}$-feines System $\mathfrak{W}=\left\{W_{1}, \ldots, W_{t}\right\}$ vom Grade $\leqslant n$, bestehend aus offenen Somen $W_{j}$ mit $Z_{j} \leqslant W_{j}(j=1, \ldots, t)$ zu konstruieren. Wir konstruieren $W_{1}$ folgendermaßen. $Z_{1}$ ist in einem Soma $U_{i} \in \mathfrak{U}$ enthalten, also fremd $\mathrm{zu} \mathrm{e} U_{i}$. Ebenso ist $Z_{1}$ fremd $\mathrm{zu}$ den endlich vielen Durchschnitten $Z_{j_{0}} \wedge \ldots \wedge Z_{j_{n}}$ mit $1<j_{0}<\ldots<j_{n}$. Also ist $Z_{1}$ fremd $\mathrm{zur}$ Vereinigung $R_{1}$ von $\mathrm{c} D_{i}$ und allen diesen Durchschnitten. $R_{1}$ ist abgeschlossen; da $\mathfrak{B}$ normal ist, existiert daher ein offenes Soma $W_{1}$ mit $Z_{1} \leqslant W_{1}$ und $\bar{W}_{1} \wedge R_{1}=O$. Dann ist $\left\{\bar{W}_{1}, Z_{2}, \ldots, Z_{t}\right\}$ ein $\mathfrak{U}$-feines System vom Grade $\leqslant n$. Analog definieren wir jetzt, ausgehend von $\left\{\bar{W}_{1}, Z_{2}, \ldots, Z_{t}\right\}$ statt von $\left\{Z_{1}, Z_{2}, \ldots, Z_{t}\right\}$, ein offenes Soma $W_{2}$ mit $Z_{2} \leqslant W_{2}$ derart, da $\beta$ $\left\{\bar{W}_{1}, \bar{W}_{2}, Z_{3}, \ldots, Z_{t}\right\}$ ein $\mathfrak{U}$-feines Somensystem vom Grade $\leqslant n$ ist. Usw. Nach $t$ Schritten ist das gewünschte Somensystem $\mathfrak{W}=\left\{W_{1}, \ldots, W_{t}\right\}$ konstruiert.

(b) Notwendig. Es sei $A$ abgeschlossen und $\mathfrak{W}=\left\{W_{1}, \ldots, W_{s}\right\}$ eine endliche, $\mathfrak{U}$-feine, offene Überdeckung des Somas $A$ vom Grade $\leqslant n$ Dann existiert eine offene Überdeckung $\left\{V_{1}, \ldots, V_{s}\right\}$ von $A$ mit $\bar{V}_{i} \leqslant W_{i}$ für jedes $i=1, \ldots, s$ (Nöbeling [3], Korollar 1 zu Satz 11.12), und das System $3=\left\{\bar{V}_{1}, \ldots, \bar{V}_{s}\right\}$ hat die verlangten Eigenschaften.

12) Für diesen Satz braucht शr nur als klassisch-topologischer normaler BooleVerband vorausgesetzt zu werden.
5.1. Voraussetzung: $-1 \leqslant n<\infty$.

Behauptung: Es ist $\operatorname{dim} \mathfrak{B} \leqslant n$, wenn und nur wenn zu jeder endli chen offenen Überdeckung $\mathfrak{U}$ von $E$ eine endliche, $\mathfrak{U}$-feine, offene Überdeckung des Somas $E$ vom Grade $\leqslant n$ existiert.

Beweis: Siehe Satz 3.9 und R. Sikorski [1] (Satz 3.5, S. 163).

Hufssatz 2. A sei ein beliebiges Soma, $-1 \leqslant n<\infty$. Ist $\operatorname{dim} A \leqslant n$, so gibt es zu jeder endlichen offenen $\ddot{U} b e r d e c k u n g ~ \mathfrak{U}=\left\{U_{1}, \ldots, U_{s}\right\}$ von $A$ eine offene Überdeckung $\left\{V_{1}, \ldots, V_{s}\right\}$ des Somas $A$ vom Grade $\leqslant n$ derart, daß $V_{i} \leqslant U_{i}$ für $i=1, \ldots, s$.

Beweis wörtlich wie Hurewicz-Wallman [1], S. $53-54$, Bem. A) u. B) sowie Theorem V. 1, wenn "set" durch "Soma" und "Decomposition Theorem" durch "Satz 3.6" ersetzt wird.

Hufssatz 3. A sei ein abgeschlossenes Soma, $-1 \leqslant n<\infty$. Ist $\operatorname{dim} A \leqslant n$, so gibt es zu jeder endlichen ofjenen $\ddot{\text { Uberdeckung }}\left\{U_{1}, \ldots, U_{m}\right\}$

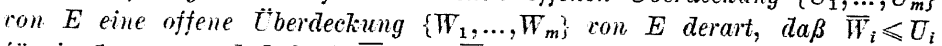
für $i=1, \ldots, m$ und daß $A \wedge \bar{W}_{i_{1}} \wedge \ldots \therefore \bar{W}_{i_{n+2}}$ leer ist für je $n+2$ verschiedene Somen $W_{i_{v}}$.

Beweis wörtlich wie K. Menger [2], S. 161, wobei der dort genannte "Hilfssatz 1" dem obigen Hilfssatz 2 entspricht.

HILFSSATZ 4. Ist $\mathfrak{F}$ ein System endlich vieler abgeschlossener Somen $F$ von endlicher Dimension, so gibt es zu jeder endlichen offenen Überdeckung $\left\{U_{1}, \ldots, U_{m}\right\}$ von $E$ eine abgeschlossene Überdeckung $\mathfrak{A}=\left\{A_{1}, \ldots, A_{m}\right\}$ von $B$ derart, daß $A_{k} \leqslant U_{k}$ für $k=1, \ldots, m$ und $d a \beta$, wenn $F$ irgend ein Soma von und $\mathfrak{A}_{r}$ irgend ein aus $r$ Somen bestehendes Untersystem von $\mathfrak{A}$ bedeutet, $\operatorname{dim} F \wedge \bigwedge_{\mathfrak{x}_{r}} A_{k} \leqslant \operatorname{dim} F-(r-1)$ ist. (Falls dabei $\operatorname{dim} F-(r-1)<-1$, so ist $\operatorname{dim} F \wedge \underset{\mathfrak{x}_{r}}{\wedge} A=-1 z u$ setzen.).

Beweis wörtlich wie K. Menger [2], S. 170-172. Dabei ist zu ersetzen: „R" durch „E"; „Hilfssatz 1' v. S. 160" durch „obiger Hilfsatz 3"; "Korollar des Summensatzes v. S. 113" durch "Satz 3.7 für $\mathfrak{B}=\mathfrak{B}_{M}$, $p=n-1, q=0$ "; "Korollar des Summensatzes v. S. 115" durch "Satz 3.4"; "Bemerkung v. S. 66" durch „Sikorski [4], S. 185"; „Bedingung c) v. S. 172",

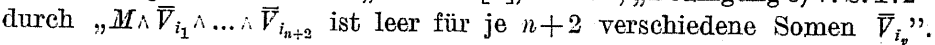

5.2. Voraussetzung: $-1 \leqslant n<\infty ; 2 \leqslant k \leqslant n+2$.

Behauptung: Es ist $\operatorname{dim} \mathfrak{B} \leqslant n$, wenn und nur wenn zu jeder endlichen offenen. Überdeckung $\mathfrak{U}$ von $E$ eine endliche, $\mathfrak{X}$-feine, abgeschlossen.e

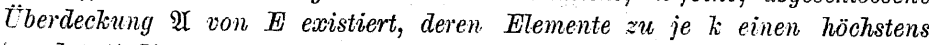
$(n-k+1)$-dimensionalen Durchschnitt besitzen.

Beweis. (a) Wenn (Menger [2], S. 181). Es sei die Bedingung des Satzes für zwei der Voraussetzung genügende, vorgelegte Zahlen $n, k$ Fundamenta Mathematicae. T. XIII. 
erfüllt. Ferner sei $\mathfrak{U}$ irgend eine endliche offene Überdeckung von $E$ und $\mathfrak{U}=\left\{A_{1}, \ldots, A_{s}\right\}$ eine endliche, $\mathfrak{U}$-feine, abgeschlossene Überdeckung von $E$, bei welcher $\operatorname{dim} \bigwedge_{\Upsilon_{i}} A_{i} \leqslant n-k+1$ ist für jedes aus $k$ Elementen bestehende Untersystem $\mathfrak{I}_{k}$ von $\mathfrak{A}$. Die Vereinigung der Durchschnitte $\bigwedge_{\mathfrak{\varkappa}_{k}} A_{i}$ für alle (endlich vielen) Untersysteme $\mathfrak{A}_{k}$ von $\mathfrak{A}$ werde mit $S_{k}$ bezeichnet. Nach 3.3 gilt dann $\operatorname{dim} S_{k} \leqslant n-k+1$; nach dem obigen Hilfssatz 2 gibt: es also eine endliche, $\mathfrak{U}$-feine, offene Überdeckung $\mathfrak{U}^{\prime}=\left\{U_{1}^{\prime}, \ldots, U_{t}^{\prime}\right\}$ des Somas $S_{k}$ vom Grade $\leqslant n-k+1$. Da $S_{k}$ abgeschlossen ist, gibt es weiter zu $\mathfrak{U}^{\prime}$ eine (endliche, $\mathfrak{U}$-feine) offene Überdeckung $\left\{W_{1}, \ldots, W_{t}\right\}$ von $S_{k}$ derart, daß $\bar{W}_{j} \leqslant U_{j}^{\prime}$ für jedes $j=1,2, \ldots, t$. Das System $\mathfrak{W}=\left\{\bar{W}_{1}, \ldots, \bar{W}_{t}\right\}$ ist $\mathfrak{U}^{\prime}$-fein und daher eine endliche, $\mathfrak{U}$-feine, abgeschlossene Überdeckung des Somas $S_{k}$ vom Grade $\leqslant n-k+1$. Setzt man nun

$$
V_{i}=A_{i} \wedge \stackrel{i}{i=1} \bar{W}_{j} \quad \text { für } \quad i=1,2, \ldots, s,
$$

so ist $\bigvee_{i=1}^{s} \bar{V}_{i} \vee \bigvee_{j=1}^{t} \bar{W}_{j}=E$; da fer̀ner $\nabla_{i} \leqslant A_{i}$, also $\bar{V}_{i} \leqslant \bar{A}_{i}=A_{i}$ und $\mathfrak{A} \mathfrak{U}$-fein ist, so ist das System $\mathfrak{A}^{\prime}=\left\{\bar{V}_{1}, \ldots, \bar{V}_{s}, \bar{W}_{1}, \ldots, \bar{W}_{t}\right\}$ eine endliche, $\mathfrak{U}$-feine, abgeschlossene Überdeckung von $E$. Wir behaupten, da $\mathfrak{U}^{\prime}$ vom Grade $\leqslant n$ ist. Sind irgend $n+2$ Elemente von $\mathfrak{U}^{\prime}$ vorgelegt, so kommen entweder mindestens $k$ der $s$ Somen $\bar{V}_{i}$ oder höchstens $k-1$ der $s$ Somen $\bar{V}_{i}$ darunter vor. Im ersten Fall ist der Durchschnitt dieser $\bar{V}_{i}$ wegen $\bar{V}_{i} \leqslant A_{i}$ in einem ${\widehat{\varkappa_{k}}}_{A_{i}}$, mithin in $S_{k}$ und daher in $\bigvee_{j=1}^{t} W_{j}$ enthalten und ist infolgedessen zu jedem $\bar{V}_{1}, \ldots, \bar{V}_{t}$ fremd; der genannte Durchschnitt ist also leer. Im zweiten Fall kommen unter den $n+2$ vorgelegten Elementen von $\mathfrak{A}^{\prime}$ mindestens $(n+2)-(k-1)=n-k+3$ der $t$ Somen $\bar{W}_{j}$ vor; deren Durchschnitt aber ist leer, weil $\mathfrak{B}$ vom Grade $\leqslant n-k+1$ war. Also ist $\mathfrak{A}^{r}$ vom Grade $\leqslant n$, wie behauptet wurde. Nach dem Hilfssatz 1 (Seite 302) gibt es nun zu $\mathfrak{U}^{\prime}$ eine endliche, $\mathfrak{H}$-feine, offene Überdeckung des Somas $E$ vom Grade $\leqslant n$, und hieraus folgt, da $\mathfrak{U}$ eine beliebige endliche offene Überdeckung von $E$ war, dim $\mathfrak{B} \leqslant n$ nach 5.1 .

(b) Nur wenn. Bezeichnet man mit $\mathfrak{F}$ das aus dem einen Soma $E$ bestehende System, so folgt aus $\operatorname{dim} E=\operatorname{dim} \mathfrak{B} \leqslant n$ die Behauptung auf Grund des obigen Hilfssatzes 4

\section{6. Trennung fremder Somen; Zusammenhang}

Wir untersuchen in diesem Paragraphen die folgenden Trennungseigenschaften eines topologischen Boole-Verbandes $\mathfrak{B}^{13}$ ):

1s) Vergl. zu (1)-(3) die Trennungsaxiome $T_{z}-T_{4}$ bei Nöbeling [3], § 11.
(3) Je zwei fremde abgeschlossene Somen sind durch ein höchstens (n-1)-dimensionales abgeschlossenes Soma getrennt; $d$. h. zu je zwei fremden abgeschlossenen Somen $A^{\prime}, A^{\prime \prime}$ gibt es ein abgeschlossenes Soma $B$ und zwei fremde offene Somen $G^{\prime}, G^{\prime \prime}$ derart, daß $\mathrm{c} B=G^{\prime} \vee G^{\prime \prime}, A^{\prime} \leqslant G^{\prime}$ $A^{\prime \prime} \leqslant G^{\prime \prime}$ und $\operatorname{dim} B \leqslant n-1$ ist.

(2) Zu je zuei fremden Somen $A^{\prime}, A^{\prime \prime}$, wo $A^{\prime}$ nicht-leer und $A^{\prime \prime}$ abgeschlossen ist, gibt es ein abgeschlossenes Soma $B$ und zwei offene Somen $G^{\prime}, G^{\prime \prime}$ derart, da $\beta$ e $B=G^{\prime} \vee G^{\prime \prime}, G^{\prime} \wedge A^{\prime}>O, A^{\prime \prime} \leqslant G^{\prime \prime}$ und $\operatorname{dim} B$ $\leqslant n-1$ ist.

(1) Zu je zwei fremden nicht-leeren Somen $\mathcal{A}^{\prime}, \mathcal{A}^{\prime \prime}$ gibt es ein abgeschlossenes Soma $B$ und zwei fremde, offene Somen $G^{\prime}, G^{\prime \prime}$ derart, daß $\mathrm{c} B$ $=G^{\prime} \vee G^{\prime \prime}, A^{\prime} \wedge G^{\prime}>0, A^{\prime \prime} \wedge G^{\prime \prime}>0$ und $\operatorname{dim} B \leqslant n-1$ ist.

Außerdem untersuchen wir die folgende Zusammenhangseigenschaft ron $\mathfrak{B}$ :

(0) E ist $(n+1)$-stufig zusammenhanglos.

Dabei wird folgendermaßen definiert:

Definition. (a) Ein Soma $A$ heiße mindestens n-stufig zusammenhängend (in $\mathfrak{B})(1 \leqslant n<\infty)$, wenn $A$ nicht-leer und kein Atom ist und wenn zu je zwei in $A$ abgeschlossenen Somen $K, L$ mit $A=K \vee L, O<K<A$ und $O<L<A$ das Soma $K \wedge L$ ein mindestens $(n-1)$-stufig zusammenhängendes Teilsoma enthält. Mindestens 0-stufig zusammenhängend heiße jedes nicht-leere Soma.

(b) Ein Soma $A$ heiße n-stufig zusammenhanglos (in $\mathfrak{B})(1 \leqslant n<\infty)$, wenn $A$ nicht-leer ist und kein (in $\mathfrak{B}$ ) mindestens $n$-stufig zusammenhängendes Teilsoma enthält. 0-stufig zusammenhanglos heiße das leere Soma und nur dieses.

Nach dieser Definition gilt offenbar Folgendes: Die mindestens 1-stufig zusammenhängenden Somen, die Atome (falls vorhanden) und das Nullsoma sind zusammenhängend. 1-stufig zusammenhanglos ist ein Soma, das nicht-leer ist und außer Atomen und dem Nullsoma keine zusammenhängenden Somen enthält, das also mit anderen Worten ,zusammenhangslos" (Menger [2], S. 202) bzw. "totally disconnected" (Hurewicz-Wallman [1], S. 15) ist.

Die Sätze dieses Paragraphen behandeln die Beziehungen der Eigenschaften (3)-(0) zueinander und zur Dimension von $\mathfrak{B}$.

6.1. Voraussetzung: $0 \leqslant n<\infty$.

Behauptung: Damit $\operatorname{dim} \mathfrak{B} \leqslant n$ sei, ist hinreichend und, falls $\mathfrak{B}$ regulär ist, auch notwendig, $d a \beta$ (2) gilt.

Beweis. (a) Hinreichend. $\mathfrak{B}$ habe die Eigenschaft (2); $\mathrm{S}$ sei ein nicht-leeres Soma und $U$ eine Umgebung von $S$. Dann ist $\mathrm{c} U$ abgeschlos- 
sen und zu $S$ fremd. Zu $A^{\prime}=S$ und $A^{\prime \prime}=\mathrm{c} U$ existiert also ein Tripel paarweise fremder Somen $B, G^{\prime}, G^{\prime \prime}$ mit den in (2) angegeben Eigenschaften. Aus $S=A^{\prime}$ folgt $S_{\wedge} G^{\prime}>O$. Wegen $\mathrm{c} U=A^{\prime \prime} \leqslant G^{\prime \prime}$ ist $\mathrm{c} G^{\prime \prime} \leqslant U$, wegen $G^{\prime} \leqslant c G^{\prime \prime}$ also $G^{\prime} \leqslant U$. Aus $\mathrm{b} G^{\prime} \leqslant B$ und $\operatorname{dim} B \leqslant n-1$ folgt $\operatorname{dim} \mathrm{b} G^{\prime} \leqslant n-1$. Also ist $\operatorname{dim} \mathfrak{B} \leqslant n$.

(b) Notwendig. Es sei dim $\mathfrak{B} \leqslant n$; ferner seien $A^{\prime}, A^{\prime \prime}$ zwei fremde Somen, $A^{\prime}$ nicht-leer und $A^{\prime \prime}$ abgeschlossen. Dann ist $U=\mathrm{c} A^{\prime \prime}$ eine Umgebung von $A^{\prime}$. Zu $A^{\prime}$ und $U$ existiert also wegen der Regularität von $\mathfrak{B}$ ein offenes Soma $U^{\prime}$ mit $\bar{U}^{\prime} \leqslant U$ und $U^{\prime} \wedge A^{\prime}>0$. Weiter gibt es wegen $\operatorname{dim} \mathfrak{B} \leqslant n$ zu $U^{\prime} \wedge A^{\prime}$ und $U^{\prime}$ ein offenes Soma $V \leqslant U^{\prime}$ mit $\nabla \wedge A^{\prime} \geqslant \nabla \wedge\left(U^{\prime} \wedge A^{\prime}\right)>0$ und $\operatorname{dim} b V \leqslant n-1$. Setzt man nun $\mathrm{b} V=B$, $V=G^{\prime}$ und $\mathrm{e} \bar{V}=G^{\prime \prime}$, so haben die Somen $B, G^{\prime}, G^{\prime \prime}$ die in (2) geforderten Eigenschaften.

6.2. Voraussetzung: $0 \leqslant n<\infty$.

Behauptung: Damit dim $\mathfrak{B} \leqslant n$ sei, ist hinreichend und, falls $\mathfrak{B}$ ein s-Verband ist, auch notwendig, daß (3) gilt.

Beweis: Satz 3.8 für $A=E$.

6.3. Voraussetzung: $0 \leqslant n<\infty$.

Behauptung: Ist $\mathfrak{B} T_{1}$-topologisch, so folgt (1) aus (2) und (2) aus (3). Ist $\mathfrak{B}$ außerdem regulär, so folgt noch (0) aus (1).

Beweis. Die erste Behauptung folgt unmittelbar daraus, daß in einem $T_{\mathbf{1}}$-topologischen Boole-Verband jedes nicht-leere Soma ein abgeschlossenes Soma $>0$ enthält.

Zum Beweis der zweiten Behauptung sei $\mathfrak{B}$ regulär und $T_{1}$-topologiseh. Es sei zunächst $n=0$ und es werde angenommen, daß $z$ war (1), aber nicht (0) gilt. Dann ist $E$ nicht 1-stufig zusammenhanglos, es gibt also ein mindestens 1 -stufig zusammenhängendes Soma A. Definitionsgemäß ist $A$ nicht-leer und kein Atom. Infolgedessen enthält $A$ ein nicht- leeres Teilsoma $A^{\prime}$ derart, daß das Soma $A^{\prime \prime}=A \wedge c A^{\prime}$ ebenfalls nicht-leer ist. Zu $A^{\prime}, A^{\prime \prime}$ existiert nun ein Tripel paarweise fremder Somen $B, G^{\prime}, G^{\prime \prime}$ mit den in (1) angegebenen Eigenschaften. Wegen $\operatorname{dim} B \leqslant-1$ ist dabei $B=O$ und folglich $A \leqslant G^{\prime} \vee G^{\prime \prime}$. Wir setzen $A \wedge G^{\prime}=K$ und $A \wedge G^{\prime \prime}=L$. Dann sind die Somen $K, L$ in $A$ abgeschlossen und nicht-leer und es ist $A=K \vee L, K<A, L<A$. Da nun $A$ mindestens 1-stufig zusammenhängend ist, muß in $K_{\wedge} L$ ein mindestens 0 -stufig zusammenhängendes, also nicht-leeres Teilsoma enthalten sein. Wegen $K_{\wedge} L \leqslant G^{\prime} \wedge G^{\prime \prime}$ steht dies aber im Widerspruch dazu, daß $G^{\prime}$ und $G^{\prime \prime}$ fremd sind. Also ist $A$ nicht mindestens 1-stufig zusammenhängend und $E$ ist in der Tat 1 -stufig
zusammenhanglos.

Nun sei die Folgerung $(1) \rightarrow(0)$ für $0 \leqslant n \leqslant m-1$ bereits bewiesen und es werde angenommen, daß für. $n=m$ zwar (1), aber nicht (0) gilt.
Dann ist $E$ nicht $(m+1)$-stufig zusammenhanglos; es gibt also ein mindestens $(m+1)$-stufig zusammenhängendes Soma $A$. $A$ ist erst recht mindestens 1-stufig zusammenhängend, also nicht-leer und kein Atom. Wie oben gibt es daher zwei nicht-leere fremde Teilsomen $A^{\prime}, A^{\prime \prime}$ von $A$, zu denen ein Tripel paarweise fremđer Somen $B, G^{\prime}, G^{\prime \prime}$ mit den in (1) angegebenen Eigenschaften existiert. Setzt man dann $\left(G^{\prime} \vee B\right) \wedge A=K$ und $\left(G^{\prime \prime} \vee B\right) \wedge A=L$, so sind die Somen $K, L$ nicht-leer und in $A$ abgeschlossen und es gilt $A=K \vee L, K<A, L<A$ sowie $K \wedge L \leqslant B$. Auss $\operatorname{dim} B \leqslant m-1$ folgt nun $\operatorname{dim} K \wedge L \leqslant m-1$; für $n=m-1$ gilt daher in dem regulären $T_{1}$-topologischen Boole-Verband $\mathfrak{B}_{K \wedge L}$ nach 6.1 die Bedingung (2) und somit auch (1). Der Induktionsvoraussetzung zufolge ist daher $K \wedge L m$-stufig zusammenhanglos und enthält also kein mindestens $m$-stufig zusammenhängendes Teilsoma. Andererseits muß aber $K \wedge L$ auf Grund der Eigenschaften von $K$ und $L$ und auf Grund dessen, daß $A$ mindestens $(m+1)$-stufig zusammenhängend ist, ein mindestens $m$-stufig $\mathrm{zu}^{-}$ sammenhängendes Teilsoma enthalten. Dies ist ein Widerspruch. Es folgt also auch für $n=m$ aus (1) die Eigenschaft (0). Damit ist 6.3 vollständig bewiesen.

Für $S$-Verbände folgt nach 6.1 und 6.2 auch (3) aus (2); die Schlüsse $(0) \rightarrow(1)$ und $(1) \rightarrow(2)$ sind aber, wie aus den bei Hurewicz-Wallman [1] (S. 20) angeführten Gegenbeispielen hervorgeht, im allgemeinen nicht richtig.

6.4. Voraussetzung: $\mathfrak{i}$ sei ein kompaleter $S$-Verband; $0 \leqslant n<\infty$,

Behauptung: Die Bedingungen (3), (2), (1), (0) und $\operatorname{dim} \mathfrak{B} \leqslant n$ sind äquivalent:

Beweis. Auf Grund der Sätze 6.2 und 6.3 genügt es nachzuweisen, daß $\mathfrak{B} \leqslant n$ aus $(0)$ folgt. Wie im Beweis von 4.6 ist $\mathfrak{B}$ atomar und homöomorph zu einem Unterverband $\mathfrak{B}^{\prime}$ eines klassisch-topologischen, kompakten, regulären $T_{1}$-Raumes $\mathfrak{E}^{\prime}$ mit abzählbarer Basis, wobei die $\Phi$-Bilder der offenen (abgeschlossenen) Somen von $\mathfrak{B}$ die offenen (abgeschlossenen) Mengen von $\mathscr{E}^{\prime}$ sind und $\operatorname{dim} A=\operatorname{dim} \Phi A$ für jedes $A \in \mathfrak{B}$ gilt.

Beachtet man nun, daß für eine beliebige nicht-leere Menge $M^{\prime} \in \mathbb{E}^{\prime}$ und für jede Zerlegung von $\bar{M}^{\prime}$ in zwei nicht-leere, abgeschlossene, echte Teilmengen $K^{\prime}, L^{\prime}$ ron $\bar{M}^{\prime}$ die Mengen $K^{\prime} \cap M^{\prime}$ und $L^{\prime} \cap M^{\prime}$ nicht-leere, in $M^{\prime}$ abgeschlossene, echte Teilmengen von $M^{\prime}$ sind, da $\beta$ infolgedessen mit $M^{\prime}$ auch $\bar{M}^{\prime}$ mindestens $k$-stufig zusammenhängend in $\mathfrak{E}^{\prime}$ ist $(k \geqslant 1)$; so ergibt sich, daß $E$ dann und nur dann $k$-stufig zusammenhanglos in $\mathfrak{V}$ ist, wenn $E^{\prime} k$-stufig zusammenhanglos in $\mathfrak{E}^{\prime}$ ist. Gilt also $(\dot{0})$. in $\mathfrak{B}$, so ist $E^{\prime}(n+1)$-stufig zusammenhanglos in E'; nàch Menger [2] (S. 213 für $n=0$, S. 216 für $n \geqslant 1$ ) folgt hieraus $\operatorname{dim} \mathbb{E}^{\prime} \leqslant n$, wegen $\operatorname{dim} \mathbb{E} \cong \operatorname{dim} E^{\prime}$ also $\operatorname{dim} \mathfrak{B} \leqslant n$, w. z. b. $w$. 


\section{7. Das Verhalten der Dimension bei Abbildungen}

7.1. Voraussetzung: $\mathfrak{B}$ und $\mathfrak{B}^{\prime}$ seien zwei $S_{-V}$-Verbände, $\Phi$ ein stetiger, abgeschlossener Vollhomomorphismus von $\mathfrak{B}$ auf $\mathfrak{B}^{\prime} ; 0 \leqslant n<\infty ; 0 \leqslant n^{\prime}<\infty$.

Behauptung: Ist $\operatorname{dim} \mathfrak{B}=n, \operatorname{dim} \mathfrak{B}^{\prime}=n^{\prime}$ und $n \leqslant n^{\prime}$, so gibt es in $\mathfrak{B}^{\prime}$ mindestens ein nicht-leeres Soma $A^{\prime}$ derart, daß im Urbild $\Phi^{-1} B^{\prime}$ eines jeden nicht-leeren Teilsomas $B^{\prime}$ von $A^{\prime}$ mindestens $n^{\prime}-n+1$ paarweise fremde, nicht-leere Somen enthalten sind.

Beweis (Menger [2], S. 237-240). Wir bezeichnen für jedes nicht. -leere Soma $A^{\prime} \in \mathfrak{B}^{\prime}$ mit $\varkappa\left(A^{\prime}\right) \leqslant \infty$ das Supremum aller Zahlen $\varkappa$ derart, daß im Urbild $\Phi^{-1} B^{\prime}$ eines jeden nicht-leeren Teilsomas $B^{\prime}$ von $A^{\prime}$ mindestens $*$ paarweise fremde, nicht-leere Teilsomen enthalten sind, und mit $k$ das Supremum der Zahlen $\varkappa\left(A^{\prime}\right)$ für alle $A^{\prime} \in \mathfrak{S}^{\prime}$. Für jedes $A^{\prime} \in \mathfrak{B}^{\prime}$ gilt dann $1 \leqslant x\left(A^{\prime}\right) \leqslant k$. Ist $k=\infty$, so ist der Satz evident. Bei endlichem $k$. besagt die Behauptung, daß $k \geqslant n^{\prime}-n+1$ oder $n^{\prime} \leqslant n+k-1$ ist; sie kamn in der letzten Form durch Induktion nach $n+k$ bewiesen werden.

Es sei $n+k=1$, also $n=0, k=1$; dann ist $n^{\prime}=0$ nachzuweisen. $A_{1}^{\prime}$ und $A_{2}^{\prime}$ seien zwei fremde abgeschlossene Somen von $\mathfrak{B}^{\prime}$. Die Somen $A_{1}=\Phi^{-1} A_{1}^{\prime}$ und $A_{2}=\Phi^{-1} A_{2}^{\prime}$ von $\mathfrak{B}$ sind dann fremd und abgeschlossen; nach 6.2 folgt daher aus $\operatorname{dim} \mathfrak{B}=0$ die Existenz zweier fremder offener Somen $G_{1}, G_{2}$ von $\mathfrak{B}$ mit $E=G_{1} \vee G_{2}, A_{1} \leqslant G_{1}$ und $A_{2} \leqslant G_{2}$. Wir betrachten nun die Somen $G_{1}^{\prime}=\Phi G_{1}$ und $G_{2}^{\prime}=\Phi G_{2}$ von $\mathfrak{V}^{\prime}$. Weil $\Phi$ abgeschlossen ist und die Somen $G_{1}, G_{2}$ in $\mathfrak{B}$ abgeschlossen sind, sind die Somen $G_{1}^{\prime}$ und $G_{2}^{\prime}$ in $\mathfrak{B}^{\prime}$ abgeschlossen. Aus $A_{1} \leqslant G_{1}$ und $A_{2} \leqslant G_{2}$ folgt $A_{1}^{\prime} \leqslant G_{1}^{\prime}$ und $A_{2}^{\prime} \leqslant G_{2}^{\prime}$. Ferner gilt $G_{1}^{\prime} \vee G_{2}^{\prime}=\Phi G_{1} \vee \Phi G_{2}=\Phi\left(G_{1} \vee G_{2}\right)=\Phi E=E^{\prime}$. Nimmt man schließlich an, es wäre $G_{1}^{\prime} \wedge G_{2}^{\prime}>O^{\prime}$, so ist $x\left(G_{1}^{\prime} \wedge G_{2}^{\prime}\right)$ definiert und $=1$ wegen $k=1$. Also existiert ein nicht-leeres Teilsoma $B^{\prime}$ von $G_{1}^{\prime} \wedge G_{2}^{\prime}$, in dessen Urbild $\Phi^{-1} B^{\prime}$ keine zwei fremden nicht-leeren Teilsomen enthalten sind. Andererseits gibt es, da $B^{\prime} \leqslant G_{1}^{\prime}=\Phi G_{1}$ und $\Phi$ ein Vollhomomorphismus ist, ein Soma $B_{1} \leqslant G_{1}$ mit $\Phi B_{1}=B^{\prime}$ and entsprechend wegen $B^{\prime} \leqslant G_{2}^{\prime}$. ein Soma $B_{2} \leqslant G_{2}$ mit $\Phi B_{2}=B^{\prime}$ (Nöbeling [3], Satz 3.6). Wegen $B^{\prime}>O^{\prime}$ ist $B_{1}>0$ und $B_{2}>O$; wegen $G_{1} \wedge G_{2}=O$ gilt $B_{1} \wedge B_{2}=O$; ferner ist $B_{1} \leqslant \Phi^{-1} B^{\prime}$ und $B_{2} \leqslant \Phi^{-1} B^{\prime}$. Das Soma $\Phi^{-1} B^{\prime}$ enthält also zwei fremde, nicht-leere men im Widerspruch zur Definition von $B^{\prime}$. Die Annahme $G_{1}^{\prime} \wedge G_{2}^{\prime}>O^{\prime}$ ist demnach falsch, es ist vielmehr $G_{1}^{\prime} \wedge G_{2}^{\prime}=O^{\prime}$. $G_{1}^{\prime}$ und $G_{2}^{\prime}$ sind infolgedessen offen und es ist $\operatorname{dim} \mathfrak{B}^{\prime} \leqslant 0$ nach 6.2 , also $\operatorname{dim} \mathfrak{B}^{\prime}=0$. Damit ist der Satz für $n+k=1$ bewiesen.

Der Satz sei nun für $1 \leqslant n+k \leqslant m-1$ bereits bewiesen und es sei $n+k=m$. Ferner seien zwei fremde abgeschlossene Somen $A_{1}^{\prime}, A_{2}^{\prime}$ von $\mathfrak{B}^{\prime}$ offene and $\operatorname{dim} B^{\prime} \leqslant m-2$ ist. Dann folgt nämlich nach $6.2 \operatorname{dim} G_{1}^{\prime} \leqslant m-1$,
1. h. $n^{\prime} \leqslant n+k-1$; der Satz gilt dann also auch für $n+k=m$, was zu zeigen ist.

Wie oben sind die Somen $A_{1}=\Phi^{-1} A_{1}^{\prime}$ und $A_{2}=\Phi^{-1} A_{2}^{\prime}$ von $\mathfrak{B}$ fremd und abgeschlossen. Wegen $\operatorname{dim} \mathfrak{B} \leqslant n$ existieren also nach 6.2 in $\mathfrak{B}$ ein abgeschlossenes Soma $B$ und zwei fremde offene Somen $G_{1}, G_{2}$ mit $\mathrm{c} B$ $=G_{1} \vee G_{2}, A_{1} \leqslant G_{1}, A_{2} \leqslant G_{2}$ und $\operatorname{dim} B \leqslant n-1$. Dabei kann ohne Beschränkung der Allgemeinheit $\bar{G}_{1} \vee \bar{G}_{2}=E$ angenommen werden (um dies zu erweisen, braucht man nur $G_{2}$ durch $e \bar{G}_{1}$ and $B$ durch $\mathrm{b} G_{1}$ zu ersetzen). Wegen $\bar{G}_{1}, \bar{G}_{2} \leqslant B$ ist $\operatorname{dim} \bar{G}_{1}, \bar{G}_{2} \leqslant n-1$. Setzt man nun

$$
\begin{gathered}
\Phi \bar{G}_{1}=M_{1}^{\prime}, \quad \Phi \bar{G}_{2}=M_{2}^{\prime}, \\
M_{1}^{\prime} \wedge M_{2}^{\prime}=B^{\prime}, \quad M_{1}^{\prime} \wedge \subset B^{\prime}=G_{1}^{\prime}, \quad M_{2}^{\prime} \wedge \subset B^{\prime}=G_{2}^{\prime},
\end{gathered}
$$

so ist $B^{\prime}$ abgeschlossen in $\mathfrak{B}^{\prime}$ und $G_{1}^{\prime}, G_{2}^{\prime}$ sind abgeschlossen in $\mathrm{c} B^{\prime}$ (weil $\Phi$ abgeschlossen ist). Weiter ist $G_{1}^{\prime} \wedge G_{2}^{\prime}=O^{\prime}$ und $G_{1}^{\prime} \vee G_{2}^{\prime}=\mathrm{c} B^{\prime}$, weil $\Phi \bar{G}_{1} \vee \Phi \bar{G}_{2}$ $=\Phi\left(\bar{G}_{1}, \bar{G}_{2}\right)=\Phi E=E^{\prime}$; die Somen $G_{1}^{\prime}, G_{2}^{\prime}$ sind folglich offen in $\mathfrak{B}^{\prime}$. Ferner gilt $A_{1}^{\prime} \leqslant G_{1}^{\prime}$, weil erstens $A_{1} \leqslant \bar{G}_{1}$, also $A_{1}^{\prime}=\Phi A_{1} \leqslant \Phi \bar{G}_{1}=M_{1}^{\prime}$, zweitens $A_{1} \wedge \bar{G}_{2}=O$, also $A_{1}^{\prime} \Phi \bar{G}_{2}=O^{\prime}$, also $A_{1}^{\prime} \wedge M_{2}^{\prime}=O^{\prime}$, und drittens $M_{1}^{\prime} \wedge \subset M_{2}^{\prime}=G_{1}^{\prime}$ ist. Schließlich gilt $A_{2}^{\prime} \leqslant G_{2}^{\prime}$ aus analogen Gründen. Wir haben jetzt also nur noch $\operatorname{dim} B^{\prime} \leqslant m-2$ zu zeigen.

Setzt man $\Phi^{-1} M_{2}^{\prime}=N$, so gilt $\left.\Phi\left(\bar{G}_{1} \wedge N\right)=\Phi \bar{G}_{1} \wedge \Phi N^{14}\right)=M_{1}^{\prime} \wedge M_{2}^{\prime}=B^{\prime}$ und $\bar{G}_{2} \leqslant N$. Das Soma $N$ von $\mathfrak{B}$ ist abgeschlossen, da $M_{2}^{\prime}$ abgeschlossen ist. Infolgedessen ist das Soma $\left(\bar{G}_{1} \wedge N\right) \wedge \mathrm{c}\left(\bar{G}_{1} \wedge \bar{G}_{2}\right)=\bar{G}_{1} \wedge N \wedge \mathrm{c} \bar{G}_{2}$ als Durchschnitt des abgeschlossenen Somas $\bar{G}_{1} \wedge N$ mit dem $F_{\sigma}$-Soma c $\bar{G}_{2}$ ein $F_{\sigma}$. Es sei etwa $\left(\bar{G}_{1} \wedge N\right) \therefore \mathrm{c}\left(\bar{G}_{1} \wedge \bar{G}_{2}\right)=\bigvee_{i=1}^{\infty} P_{i}$, also $\bar{G}_{1} \wedge N=\left(\bar{G}_{1} \wedge \bar{G}_{2}\right) \cup \bigvee_{i=1}^{\infty} P_{i}$, wo $P_{i}$ für $i=1,2, \ldots$ abgeschlossen ist. Aus der zweiten Beziehung folgt wegen $\Phi\left(\bar{G}_{1} \wedge N\right)=B^{\prime}$

$$
B^{\prime}=\Phi\left(\bar{G}_{1} \wedge \bar{G}_{2}\right) \cup \bigvee_{i=1}^{\infty} \Phi P_{i}
$$

wobei jeder Summand der rechten Seite als Bild eines abgeschlossenen Somas selbst abgeschlossen ist.

Der Vollhomomorphismus $\Phi$, nur auf dem $S$-Verband $\mathfrak{B}_{\bar{G}_{1 /}, \bar{G}_{2}}$ betrachtet, ist ein Vollhomomorphismus von $\mathfrak{B}_{\bar{G}_{1}: \bar{G}_{2}}$ auf den $S$-Verband $\mathfrak{B}_{\Phi\left(\bar{G}_{1} \wedge \bar{G}_{2}\right)}^{\prime}$. Da $\operatorname{dim} \bar{G}_{1} \wedge \bar{G}_{2} \leqslant n-1$ ist, ergibt die Induktionsvoraussetzung $\operatorname{dim} \Phi\left(\bar{G}_{1} \bar{\wedge} \bar{G}_{2}\right) \leqslant m-2$.

Wir betrachten nun ein Soma $P_{i}$. Ist $P_{i}=O$, so ist $\Phi P_{i}=O^{\prime}$ und daher $\operatorname{dim} \Phi P_{i} \leqslant m-2$. Nun sei $P_{i}>0$ (also $\Phi P_{i}>O^{\prime}$ ). Wir behaupten wieder $\operatorname{dim} \Phi P_{i} \leqslant m-2$. Hierzu betrachten wir den Vollhomomorphismus $\Phi$ nur auf dem $S$-Verband $\mathfrak{M}=\mathfrak{B}_{P_{i}}$; er ist ein Vollhomomorphismus von $\mathfrak{W}$ auf den $S$-Verband $\mathfrak{W}^{\prime}=\mathfrak{B}_{\Phi\left(P_{i}\right)}^{\prime}$. Es ist $\operatorname{dim} \mathfrak{W} \leqslant n$; die $\mathfrak{W}$ und $\mathfrak{W}^{\prime}$

14) Nöbeling [3], Satz 3.5. 
entsprechende Zahl $k$, wir bezeichnen sie mit $l$, ergibt sich aus den folgenden Überlegungen. Aus $P_{i} \leqslant \bar{G}_{1} \wedge N \wedge \mathrm{c} \bar{G}_{2}$ folgt $P_{i} \wedge \bar{G}_{2}=O$ sowie $P_{i} \leqslant N$, also $\Phi P_{i} \leqslant \Phi N=M_{2}^{\prime}=\Phi \bar{G}_{2}$. Bestünde nun für ein nicht-leeres Teilsoma $S^{\prime}$ von $\Phi P_{i}$ die Gleichung $\bar{G}_{2} \wedge \Phi^{-1} S^{\prime}=0$, so wäre

$$
\Phi \bar{G}_{2} \wedge S^{\prime}=\Phi \bar{G}_{2} \wedge \Phi \Phi^{-1} S^{\prime}=\Phi\left(\bar{G}_{2} \wedge \Phi^{-1} S^{\prime}\right)=\Phi O=0^{\prime}
$$

im Widerspruch zur Relation $S^{\prime} \leqslant \Phi P_{i} \leqslant \Phi \bar{G}_{2}$. Infolgedessen gilt für jedes nicht-leere Teilsoma $S^{\prime}$ von $\Phi P_{i}$ die Beziehung $\Phi^{-1} S^{\prime} \wedge \bar{G}_{2}>O$, während $P_{i} \wedge \bar{G}_{2}=O$ ist. Die beiden letzten Relationen besagen nun, daß das Urbild $\Phi^{-1} S^{\prime}$ eines jeden nicht-leeren Teilsomas $S^{\prime}$ von $\Phi P_{i}$ ein zu $P_{i}$ fremdes, nicht-leeres Teilsoma enthält. Hieraus folgt $l \leqslant k-1$. Wir können daher die Induktionsvoraussetzung auf die Verbände $\mathfrak{B}$ und $\mathfrak{W}^{\prime}$ anwenden nnd erhalten $\operatorname{dim} \Phi P_{i} \leqslant m-2$.

Es ist somit $B^{\prime}$ die Vereinigung abzählbar vieler, in $\mathfrak{V}^{\prime}$ und daher in $B^{\prime}$ abgeschlossener, höchstens $(m-2)$-dimensionaler Somen; nach 3.3 gilt also $\operatorname{dim} B^{\prime} \leqslant m-2$, was zu zeigen war:

7.2. Voraussetzung: $\mathfrak{B}$ und $\mathfrak{B}^{\prime}$ seien zwei kompakte S-Verbände, $\Phi$ ein stetiger, abgeschlossener Vollhomomorphismus von $\mathfrak{B}$ auf $\mathfrak{B}^{\prime}, 0 \leqslant n<\infty$, $0 \leqslant n^{\prime}<\infty$.

Behauptung: Ist $\operatorname{dim} \mathfrak{B}=n, \operatorname{dim} \mathfrak{V}^{\prime}=n^{\prime}$ und $n \geqslant n^{\prime}$, so gibt es in $\mathfrak{V}^{\prime}$ mindestens ein nicht-leeres Soma $A^{\prime}$ derart, ḋa für jedes nicht-leere Teitsoma $B^{\prime}$ von $A^{\prime} \operatorname{dim} \Phi^{-1} B^{\prime} \geqslant n-n^{\prime}$ ist.

Beweis. Wie im Beweis von 4.6. ist $\mathfrak{B}$ atomar und homöomorph zu einem Unterverband eines klassisch-topologischen, kompakten, regulären $T_{1}$-Raumes mit abzählbarer Basis: Identifiziert man die Somen von $\mathfrak{B}$ mit ihren Bildern in diesem Raum, so ist $\mathfrak{B}$ Unterverband eines klassisch-topologischen, kompakten, regulären $T_{1}$-Raumes E mit abzählbarer Basis derart, daß die offenen (abgeschlossenen) Somen von $\mathfrak{B}$ die offenen (abgeschlossenen) Mengen von $\mathbb{E}$ sind und daß für jedes Soma von $\mathfrak{B}$ die in $\mathfrak{P}$ genommene Dimension gleich der in $\mathfrak{E}$ genommenen ist. Entsprechend ist $\mathfrak{B}^{\prime}$ Unterverband eines Raumes $\mathbb{E}^{\prime}$ mit den gleichen Eigenschaften. Durch die im vorliegenden Satz vorausgesetzte Abbildung $\Phi$ ist nun jedem Atom $P$ von $\mathfrak{B}$ (also von $\mathfrak{F}$ ) eindeutig ein Atom $\Phi P$ von $\mathfrak{B}^{\prime}$ (also von $\mathfrak{E}^{\prime}$ ), und jedem Soma $A$ von $\mathfrak{B}$ die Menge $\Phi_{A}$ aller Atome $\Phi P$ von $\mathfrak{B}^{\prime}$ mit $P \leqslant A$ zugeordnet. Ordnet man daher jeder Menge $M \in \mathbb{E}$ die Menge $\Psi M$ aller Atome $\Phi P \in \mathbb{E}^{\prime}$ mit $P \leqslant M$ zu, so ist $\Psi$ ein stetiger, abgeschlossener Vollhomomorphismus von $\mathbb{E}$ auf $\mathbb{E}^{\prime}$ derart, daß $\Psi A=\Phi A$ für jedes Soma $A \in \mathfrak{B}$ und $\Psi^{-1} A^{\prime}=\Phi^{-1} A^{\prime}$ für jedes $A^{\prime} \in \mathfrak{V}^{\prime}$ ist. Nun ist $\operatorname{dim} \mathbb{E}=n, \operatorname{dim} \mathbb{E}^{\prime}=n^{\prime}$ und $n \geqslant n^{\prime}$. Nach K. Menger [2] (S. 235) gibt es daher mindestens ein Atom $P^{\prime} \in \mathbb{E}^{\prime}$ mit $\operatorname{dim} \Psi^{-1} P^{\prime}$ $\geqslant n-n^{\prime}$ (diese Dimension in E genommen). Weil $P^{\prime}$ zu $\mathfrak{V}^{\prime}$ gehört, ist
$\Psi^{-1} P^{\prime}=\Phi^{-1} P^{\prime}$ und $\operatorname{dim} \dot{\Phi}^{-1} P^{\prime} \geqslant n-n^{\prime}$ (diese Dimension in $\mathfrak{B}$ genommen). Da $P^{\prime}$ kein nicht-leeres echtes Teilsoma enthält, leistet $P^{\prime}$ das in 7.2 Verlangte.

\section{Zitate}

[1] W. Hurewicz and H. Wallman, Dimension theory, Princeton 1948.

[2] K. Menger, Dimensionstheorie, Leipzig 1928.

[3] G. Nöbeling, Grundlagen der Analytischen Topologie, Heidelberg-GöttingenBerlin 1954.

[4] R. Sikorski, Closure algebras, Fund. Math. 36 (1949), p. 165-206.

[5] - Dimension theory in closure algebras, Fund. Math. 38 (1951), p. 153-166.

Reçu par la Rédaction le 15.10.1954 